\title{
Strongly Graded C*-algebras
}

by

Ellis Dawson

\author{
A thesis \\ submitted to the Victoria University of Wellington \\ in fulfilment of the \\ requirements for the degree of \\ Master of Science \\ in Mathematics.
}

Victoria University of Wellington

2020 



\begin{abstract}
We investigate strongly graded $C^{*}$-algebras. We focus on graph $C^{*}$-algebras and explore the connection between graph $C^{*}$-algebras and Leavitt path algebras, both of which are $\mathbb{Z}$-graded. It is known that a graphical condition called Condition $(Y)$ is necessary and sufficient for Leavitt path algebras to be strongly graded. In this thesis we prove this can be translated to the graph $C^{*}$-algebra and prove that a graph $C^{*}$-algebra associated to a row-finite graph is strongly graded if and only if Condition ( $\mathrm{Y}$ ) holds.
\end{abstract}




\section{Acknowledgments}

I would like to express my deep gratitude to Lisa and Iain, my research supervisors, for their patient guidance, enthusiastic encouragement and useful critiques of this research. 


\section{Contents}

1 Introduction $\quad 1$

2 Directed Graphs And Their Algebras 5

2.1 Directed Graphs . . . . . . . . . . . . . . . . 5

2.2 Leavitt Path Algebras . . . . . . . . . . . . . . 6

2.3 Graph $C^{*}$-algebras . . . . . . . . . . . . . . . 11

3 Gradings 17

3.1 The $\mathbb{Z}$-grading of $L_{\mathbb{C}}(E) \ldots \ldots \ldots \ldots \ldots$

3.2 The $\mathbb{Z}$-grading of Graph $C^{*}$-algebras . . . . . . . . . . . 25

3.3 The Core . . . . . . . . . . . . . . . . . 37

3.4 Fell Bundles . . . . . . . . . . . . . . . . . . 40

4 Strongly Graded Graph C*-algebras 49 


\section{Chapter 1}

\section{Introduction}

We say that a ring $A$ is graded by a group $G$ if $A=\bigoplus_{g \in G} A_{g}$, where each $A_{g}$ is an additive subgroup of $A$, and $A_{g} \cdot A_{h} \subseteq A_{g h}$ for every $g, h \in G$. For a $G$-graded ring $A$ every element $a \in A$ has a unique decomposition $a=\sum_{g \in F} a_{g}$ where each $a_{g} \in A_{g}$ and $F$ is a finite subset of $G$. A motivating example is a polynomial ring, which is graded by the integers, $\mathbb{Z}$.

In a recent book [5], Exel defines a $C^{*}$-algebra to be $G$-graded if it contains a dense $G$-graded ring. In fact, Exel also introduces a stronger notion: a $G$-graded $\mathrm{C}^{*}$-algebra is topologically graded if there is a bounded linear map $F: A \rightarrow A$ which is the identity on $A_{e}$ and vanishes on every $A_{g}$ with $g \neq e$ [5, Definition 19.2]. Results from both [5] and [12] demonstrate that there is an interesting relationship between the algebraic theory of graded rings and analytic theory of graded $C^{*}$-algebras. Expressly, each grading subspace of a graded $\mathrm{C}^{*}$-algebra is a Banach space and the collection of these form an analytic structure known as a Fell bundle.

We say that a ring is strongly graded if in addition to being $G$-graded, $A_{g} \cdot A_{h}=A_{g h}$ for every $g, h \in G$. In [4], Dade systematically studied strongly graded rings and claimed that "their gradings alone are their most important property".

In this thesis we aim to introduce the notion of strongly graded $\mathrm{C}^{*}$ - 
algebras. In the literature, a Fell bundle is called saturated if it satisfies a condition which looks strikingly similar to the condition for strong grading. We expect the relationship between strong grading and saturated Fell bundles to reflect the relationship between grading and Fell bundles.

We focus our investigation within the specific setting of graph rings and algebras. To any directed graph we can associate both a purely algebraic ring called the Leavitt path algebra as well as a $C^{*}$-algebra such that the Leavitt path algebra sits densely inside the graph $C^{*}$-algebra. The connection between the two have captured the attention of ring theorists and analysts alike.

Both the graph $C^{*}$-algebra and the Leavitt path algebra are graded by $\mathbb{Z}$. In the preprint [3], a graphical condition is given such that a graph ring is strongly graded if and only if the condition is met. The main goal of this thesis is to demonstrate how this characterisation gives similar results for graph $C^{*}$-algebras by showing that its grading generates a saturated Fell bundle.

Numerous results have been found wherein the Leavitt path algebra has a particular property if and only if the graph $C^{*}$-algebra shares said property (or some topological equivalent) and our main theorem fits this model. Typically the arguments for these results run parallel to one another; it is shown that a Leavitt path algebra has a property if and only if the associated graph has some property, and the same is proven for the graph $\mathrm{C}^{*}$-algebra using different techniques. The link between the graph $C^{*}$-algebra and the Leavitt path algebra is only made at the level of the graph. The novelty of our approach is that we deduce our $C^{*}$-algebra result directly from the analogous result about Leavitt path algebras.

We assume a basic understanding of $C^{*}$-algebras, though we will define the terminologies when needed including the definitions and conventions of graph $C^{*}$-algebras which follow [10].

We now outline the structure of this thesis. In the second chapter we 
define and review some of the basic properties of Leavitt path algebras and graph $\mathrm{C}^{*}$-algebras. The third chapter discusses grading in both the algebraic and the $\mathrm{C}^{*}$-algebraic setting; in particular, we will show that both the Leavitt path algebra and the graph $C^{*}$-algebra are graded by the group $\mathbb{Z}$. We also look at the structure of the 0 -graded piece, $A_{0}$, of both structures. We show that the 0 -graded piece of the Leavitt path algebra and the graph $C^{*}$-algebra are isomorphic to the direct limit of matrix algebras. Finally, in Chapter 3 we examine Fell bundles over $C^{*}$-algebras, which we will see are intrinsically tied to $C^{*}$-grading, and present some properties of saturated Fell bundles. These chapters will cover most of the preliminary knowledge required.

In the fourth chapter we introduce the notion of a strongly graded $\mathrm{C}^{*}$ algebra and present our main result. Namely, we prove that the $C^{*}$-algebra of a row-finite directed $E$ graph is strongly graded if and only if $E$ has no sources and satisfies Condition (Y). 


\section{Chapter 2}

\section{Directed Graphs And Their Algebras}

In this chapter we discuss directed graphs and their associated algebras. We begin by introducing directed graphs and terms used to describe their components and properties. Then for a directed graph $E$ we discuss the associated Leavitt path algebra ring $L_{K}(E)$, where $K$ is an arbitrary field, which is built using an axiomatised set of relations over the vertices and edges of the graph. We then move on to discussing Cuntz-Krieger Efamilies which we use to establish the graph $C^{*}$-algebra $C^{*}(E)$. We show that the two structures are founded upon similar relations, and are strongly connected. In fact, there is a dense Leavitt path algebra $L_{\mathbb{C}}(E)$ embedded in $C^{*}(E)$, which we use to form the crucial argument for the main result of this thesis.

\subsection{Directed Graphs}

A directed graph $E$ is a quadruple $\left(E^{0}, E^{1}, r, s\right)$, consisting of two countable sets, $E^{0}, E^{1}$ and two functions $r, s: E^{1} \rightarrow E^{0}$. We call $E^{0}$ the set of vertices of $E$ and $E^{1}$ the set of edges of $E$. For an edge $e \in E^{1}$ we call $s(e)$ the source of $e$, and $r(e)$ the range of $e$. If $e \in E^{1}$ such that $s(e)=v$ and $r(e)=w$ we 
say that $v$ emits $e$ and that $w$ receives $e$. For the purposes of this thesis all graphs will be directed unless otherwise stated so the reader may always assume the term "graph" refers to directed graphs as defined above.

An edge with the same source and range is known as a loop. A vertex which emits no edge is known as a sink and a vertex that receives no edge is known as a source (the double up in terminology does not seem to cause any ambiguity within the literature).

It is often useful to represent graphs by drawing them; however, there are many ways to draw a graph. It is important to note that two graphs $E$ and $F$ are isomorphic if and only if there are two bijective functions $\phi^{0}$ : $E^{0} \rightarrow F^{0}$, and $\phi^{1}: E^{1} \rightarrow F^{1}$ such that $r_{F} \circ \phi^{1}=\phi^{0} \circ r_{E}$ and $s_{F} \circ \phi^{1}=\phi^{0} \circ s_{F}$.

We say that a graph $E$ is row-finite if the vertex matrix $A_{E}$ of the graph $E$, which is the $E^{0} \times E^{0}$ matrix with entries

$$
A_{E}(v, w)=\#\left\{e \in E^{1}: r(e)=v, s(e)=w\right\}
$$

is such that each row has a finite sum.

\subsection{Leavitt Path Algebras}

Let $E$ be a row-finite directed graph and $K$ be any field. Define the set $\left(E^{1}\right)^{*}:=\left\{e^{*}: e \in E^{1}\right\}$ to be a new set of edges consisting of the edges of $E$ with the direction reversed so that $s\left(e^{*}\right)=r(e)$ and $r\left(e^{*}\right)=s(e)$. We sometimes refer to these as ghost edges. The Leavitt path algebra $L_{K}(E)$ is the free associative $K$-algebra generated by $E^{0} \cup E^{1} \cup\left(E^{1}\right)^{*}$ subject to the following relations:

1. For all $v, w \in E^{0}, v w=\delta_{v, w} v$;

2. For all $e \in E^{1}, e=r(e) e=e s(e)$;

3. For all $e \in\left(E^{1}\right)^{*}, e^{*}=s(e) e^{*}=e^{*} r(e)$; 
4. For all $e, f \in E^{1}, e^{*} f=\delta_{e, f} s(e)$;

5. For all $v \in E^{0}, v=\sum_{\left\{e \in E^{1}: r(e)=v\right\}} e e^{*}$ whenever $v$ is not a source.

These relations can vary within the literature where $r$ and $s$ are essentially swapped and sinks become sources and vice versa. The above definition and the results in the rest of this section can be found in [1, Chapter 1, Section 2] with $r$ and $s$ interchanged. We have chosen this convention to be consistent with [10]. This will mean that the paths of a graph will appear to be in reverse; however, this approach is better suited for the context of higher rank graphs.

Remark 2.2.1 (The Universal Property of $L_{K}(E)$ ). Leavitt path algebras are universally defined in the following sense: Suppose $E$ is a graph, and $A$ is a $K$-algebra which contains a set of pairwise orthogonal idempotents $\left\{a_{v}: v \in E^{0}\right\}$, and two sets $\left\{a_{e}: e \in E^{1}\right\}$, and $\left\{b_{e}: e \in E^{1}\right\}$ for which

(i) $a_{e}=a_{r(e)} a_{e}=a_{e} a_{s(e)}$ and $b_{e}=a_{s(e)} b_{e}=b_{e} a_{r(e)}$ for all $e \in E^{1}$;

(ii) $b_{e} a_{f}=\delta_{e, f} a_{s(e)}$ for all $e, f \in E^{1}$; and

(iii) $a_{v}=\sum_{\left\{e \in E^{1}: r(e)=v\right\}} a_{e} b_{e}$ whenever $v$ is not a source;

then there exists a unique $K$-algebra homomorphism $\phi: L_{K}(E) \rightarrow A$ such that $\phi(v)=a_{v}, \phi(e)=a_{e}$ and $\phi\left(e^{*}\right)=b_{e}$ for all $v \in E^{0}$ and $e \in E^{1}[1$, Remark 1.2.5]. We call such a family a Leavitt E-family in $A$.

We now give some basic properties of $L_{K}(E)$. The following calculations and terminologies have been adapted from chapter one of [10] for Leavitt E-families in order to give a more detailed proof of [1, Lemma 1.2.12].

Proposition 2.2.2. Let $E$ be a row-finite graph, let $K$ be a field. Then for $e, f \in$ $L_{K}(E)$, we have that:

(i) ef $\neq 0 \Longrightarrow s(e)=r(f)$; 
(ii) $e^{*} f^{*} \neq 0 \Longrightarrow r(e)=s(f)$;

(iii) $e f^{*} \neq 0 \Longrightarrow s(e)=s(f)$;

(iv) $\left(e e^{*}\right)\left(f f^{*}\right)= \begin{cases}e e^{*} & \text { if } e=f \\ 0 & \text { else. }\end{cases}$

Proof. To begin with fix $e, f \in E^{0}$. To prove $(i)$, notice that

$$
\begin{array}{rlrl}
e f & =(e s(e))(r(e) f) & & \text { by the second relation for } L_{K}(E) \\
& =e(s(e) r(f)) f & \\
& =e\left(\delta_{s(e), r(f)} s(e)\right) f, & & \text { by the first relation for } L_{K}(E)
\end{array}
$$

and

$$
\begin{aligned}
\delta_{s(e), r(f)} s(e) & \neq 0 \\
& \Longrightarrow s(e)=r(f)
\end{aligned}
$$

according to the first Leavitt path algebra relations; giving us $(i)$. We can use a similar calculation prove $(i i)$ and $(i i i)$.

Lastly, for $(i v),\left(e e^{*}\right)\left(f f^{*}\right)=e\left(e^{*} f\right) f^{*}=e\left(\delta_{e, f} s(e)\right) f^{*}$ by the fourth Leavitt path algebra relation. So either

$$
\left(e e^{*}\right)\left(f f^{*}\right)=e 0 f^{*}=0
$$

implying $e \neq f$; else $e=f$ and

$$
\left(e e^{*}\right)\left(f f^{*}\right)=e\left(e^{*} e\right) e^{*}=e s(e) e^{*}=e e^{*} .
$$

Part $(i)$ of Proposition 2.2.2 is particularly useful because this allows us to think of paths as elements of the Leavitt path algebra. A path of length $n$ in a directed graph $E$ is a sequence of edges $\mu=\mu_{1} \cdots \mu_{n}$ in $E$ such that $s\left(\mu_{i}\right)=r\left(\mu_{i+1}\right)$ for $i \in\{1, \ldots, n-1\}$. We denote the length of $\mu$ as 
$|\mu|:=n$, and consider vertices to be paths of length 0 . We say that $E^{n}$ is the set of paths of length $n$ in $E$. We call the set of all paths of a graph $E^{*}:=\bigcup_{n \geq 0} E^{n}$. We can extend the range and source maps to paths of $E$ by setting $r(\mu)=r\left(\mu_{1}\right)$ and $s(\mu)=s\left(\mu_{|\mu|}\right)$ for $\mu \in E^{*}$. For $\mu, \nu \in E^{*}$ with $s(\mu)=r(\nu)$, we write $\mu \nu$ for the path $\mu_{1} \cdots \mu_{|\mu|} \nu_{1} \cdots \nu_{|\nu|}$.

When we view a finite sequence of edges as a product of edges in the Leavitt path algebra Proposition 2.2.2 tells us that for any product of length $n, \mu=\mu_{1} \cdots \mu_{n}=0$ unless $\mu$ is a path. If $\mu$ is a path then:

$$
\begin{aligned}
\mu^{*} \mu & =\left(\mu_{1} \cdots \mu_{n}\right)^{*} \mu_{1} \cdots \mu_{n} \\
& =\mu_{n}^{*} \cdots \mu_{2}^{*}\left(\mu_{1}^{*} \mu_{1}\right) \mu_{2} \cdots \mu_{n} \\
& \left.=\mu_{n}^{*} \cdots \mu_{2}^{*} s\left(\mu_{1}\right) \mu_{2} \cdots \mu_{n} \quad \text { (fourth relation for } L_{K}(E)\right) \\
& =\mu_{n}^{*} \cdots \mu_{2}^{*} r\left(\mu_{2}\right) \mu_{2} \cdots \mu_{n} \quad\left(s\left(\mu_{i}\right)=r\left(\mu_{i+1}\right)\right) \\
& =\mu_{n}^{*} \cdots \mu_{3}^{*}\left(\mu_{2}^{*} \mu_{2}\right) \mu_{3} \cdots \mu_{n} \\
& \vdots \\
& =s\left(\mu_{n}\right)=s(\mu) .
\end{aligned}
$$

We can extend the results of Proposition 2.2.2 in the following way:

Corollary 2.2.3. Let $E$ be a row-finite directed graph and $K$ be a field. Then for paths $\mu, \nu \in E^{*}$ in the Leavitt path algebra $L_{K}(E)$ we have that:

(i) $\mu \nu \neq 0 \Longrightarrow \mu \nu \in E^{*}$;

(ii) $\mu \nu^{*} \neq 0 \Longrightarrow s(\mu)=s(\nu)$;

(iii) $\mu^{*} \nu^{*} \neq 0 \Longrightarrow \nu \mu \in E^{*}$;

(iv) if $|\mu|=|\nu|$ and $\mu \neq \nu$, then $\left(\mu \mu^{*}\right)\left(\nu \nu^{*}\right)=0$;

(v) $\mu^{*} \nu= \begin{cases}\mu^{\prime *} & \text { if } \mu=\nu \mu^{\prime} \text { for some } \mu^{\prime} \in E^{*} \\ \nu^{\prime} & \text { if } \nu=\mu \nu^{\prime} \text { for some } \nu^{\prime} \in E^{*} . \\ 0 & \text { else }\end{cases}$ 
Proof. Fix $\mu, \nu \in E^{*}$. Firstly, we show $(i)$. As discussed above $\mu \nu=$ $\mu_{1} \cdots \mu_{|\mu|} \nu_{1} \cdots \nu_{|\nu|}$. Suppose $\mu \nu \neq 0$, then

$$
\begin{aligned}
\mu_{|\mu|} \nu_{1} \neq 0 & \Longrightarrow s\left(\mu_{|\mu|}\right)=r\left(\nu_{1}\right) \quad \text { by Proposition 2.2.2.(i) } \\
& \Longrightarrow \mu \nu \in E^{*}
\end{aligned}
$$

Similarly for $(i i)$, we can see that

$$
\begin{aligned}
\mu \nu^{*} & =\mu_{1} \cdots \mu_{|\mu|}\left(\nu_{1} \cdots \nu_{|\nu|}\right)^{*} \\
& =\mu_{1} \cdots \mu_{|\mu|} \nu_{|\nu|}^{*} \cdots \nu_{1}^{*} .
\end{aligned}
$$

If $\mu \nu^{*} \neq 0$ then $\mu_{|\mu|} \nu_{|\nu|} \neq 0$ which implies $s\left(\mu_{|\mu|}\right)=s\left(\nu_{|\nu|}\right)$ by Proposition 2.2.2. (iii), and thus $s(\mu)=s\left(\mu_{|\mu|}\right)=s\left(\nu_{|\nu|}\right)=s(\nu)$.

To see $(i i i)$, notice $\mu^{*} \nu^{*}=(\nu \mu)^{*}$, which is non-zero by $(i)$ whenever $\nu \mu \in E^{*}$.

To see $(i v)$, suppose $|\mu|=|\nu|=n$ and let $i$ be the smallest integer such that $\mu_{i} \neq \nu_{i}$. Then

$$
\begin{array}{rlrl}
\mu^{*} \nu & =\left(\mu_{1} \cdots \mu_{n}\right)^{*} \nu_{1} \cdots \nu_{n} & \\
& =\mu_{n}^{*} \cdots \mu_{i}^{*}\left(\mu_{i-1}^{*} \cdots \mu_{1}^{*}\right)\left(\nu_{1} \cdots \nu_{i-1}\right) \nu_{i} \cdots \nu_{n} & \\
& =\mu_{n}^{*} \cdots \mu_{i}^{*} s\left(\mu_{i-1}\right) \nu_{i} \cdots \nu_{n} & & \text { by Equation }(2.1) \\
& =\mu_{n}^{*} \cdots \mu_{i}^{*} r\left(\mu_{i}\right) \nu_{i} \cdots \nu_{n} & & \text { as } s\left(\mu_{i-1}\right)=r\left(\mu_{i}\right) \\
& =\mu_{n}^{*} \cdots \mu_{i}^{*} \nu_{i} \cdots \nu_{n} & & \text { by fourth relation for } L_{K}(E) .
\end{array}
$$

Which is equal to 0 since $\mu_{i}^{*} \neq \nu_{i}$ by fourth relation for Leavitt path algebras.

Finally, for $(v)$ suppose that $|\mu|=n$, and $|\mu| \leq|\nu|$. Let $\nu=\alpha \nu^{\prime}$ with $|\alpha|=n$. Then $\mu^{*} \nu=\mu^{*}\left(\alpha \nu^{\prime}\right)=\left(\mu^{*} \alpha\right) \nu^{\prime}$. If $\mu=\alpha$, then by Equation (2.1) $\left(\mu^{*} \alpha\right) \nu^{\prime}=s(\mu) \nu^{\prime}$. Else, $\mu \neq \alpha$ and $\left(\mu^{*} \alpha\right) \nu^{\prime}=0 \nu^{\prime}=0$ by $(i)$. In the case where $|\mu|>|\nu|$ we can show $(v)$ using a similar argument factoring $\mu=$ $\beta \mu^{\prime}$.

We are now able to prove the following lemma which can be found in [1, Lemma 1.2.12]. 
Lemma 2.2.4. Let $E$ be a row-finite graph and $K$ be a field. Then for paths $\mu, \nu, \alpha, \beta \in E^{*}$ in $L_{K}(E)$ we have

$$
\left(\mu \nu^{*}\right)\left(\alpha \beta^{*}\right)= \begin{cases}\left(\mu \alpha^{\prime}\right) \beta^{*} & \text { if } \alpha=\nu \alpha^{\prime} \text { for some } \alpha^{\prime} \in E^{*} \\ \mu\left(\beta \nu^{\prime}\right)^{*} & \text { if } \nu=\alpha \nu^{\prime} \text { for some } \nu^{\prime} \in E^{*} \\ 0 & \text { else. }\end{cases}
$$

In particular, the algebra $L_{K}(E)$ is spanned as a $K$-vector space by the set

$$
\left\{\mu \nu^{*}: \mu, \nu \in E^{*}, s(\mu)=s(\nu)\right\} .
$$

Proof. Fix $\mu, \nu, \alpha, \beta \in E^{*}$. If $s(\mu) \neq s(\nu)$ or $s(\alpha) \neq s(\beta)$, then $\left(\mu \nu^{*}\right)\left(\alpha \beta^{*}\right)=0$ by Corollary 2.2.3.(ii). Suppose that $s(\mu)=s(\nu)$ and $s(\alpha)=s(\beta)$. Then

$$
\left(\mu \nu^{*}\right)\left(\alpha \beta^{*}\right)=\mu\left(\nu^{*} \alpha\right) \beta^{*}= \begin{cases}\mu \alpha^{\prime} \beta^{*} & \text { if } \alpha=\nu \alpha^{\prime} \text { for some } \alpha^{\prime} \in E^{*} \\ \mu\left(\beta \nu^{\prime}\right)^{*} & \text { if } \nu=\alpha \nu^{\prime} \text { for some } \nu^{\prime} \in E^{*} \\ 0 & \text { else }\end{cases}
$$

by Corollary 2.2.3. $(v)$.

To show the second statement of this proposition, take a non-zero string of elements $e \in E^{1}$ and $f^{*} \in\left(E^{1}\right)^{*}$. Adjacent $e^{\prime}$ s can be written as a path $\mu \in E^{*}$ and adjacent $f^{* \prime}$ s can be written as $\nu^{*}$ for some $\nu \in E^{*}$. So the string is a product of terms of the form $\mu \nu^{*}$; for example we can write $\mu^{*}$ as $s(\mu) \mu^{*}$. Equation (2.2) tells us a product of these terms can be simplified to a single term of that form. Thus, every element of $L_{K}(E)$ is a sum of terms of the form $\mu \nu^{*}$ and $L_{K}(E)=\operatorname{span}\left\{\mu \nu^{*}: \mu, \nu \in E^{*}, s(\mu)=s(\nu)\right\}$.

\subsection{Graph $C^{*}$-algebras}

We now seek to represent a directed graph $E$ with operators on a Hilbert space $\mathcal{H}$. When building a graph $\mathrm{C}^{*}$-algebra from a graph we represent the vertices with projective subspaces of $\mathcal{H}$ and the edges with partial isometries mapping between them. It is important to note that there is 
no standard method to prescribing which direction to put the isometries. Some authors will put them in the same direction as the edges, and others will put them in the opposite direction. Each method comes with its own advantages and disadvantages. A large majority of the information regarding graph $\mathrm{C}^{*}$-algebras in this thesis, the remainder of this chapter in particular, is from [10] and as such we will be using the conventions used by Raeburn.

Let $E$ be a row-finite directed graph and $\mathcal{H}$ be a Hilbert space. A CuntzKrieger E-family $\{S, P\}$ on $\mathcal{H}$ consists of a set of mutually orthogonal projections $\left\{P_{v}: v \in E^{0}\right\}$ and a set $\left\{S_{e}: e \in E^{1}\right\}$ of partial isometries on $\mathcal{H}$ such that

$(\mathrm{CK} 1) S_{e}^{*} S_{e}=P_{v}$

(CK2) $P_{v}=\sum_{e \in E^{1}: r(e)=v} S_{e} S_{e}^{*}$ whenever $v$ is not a source.

These conditions are known as Cuntz-Krieger relations. In particular, $\mathrm{CK}(2)$ is known as the Cuntz-Krieger relation at $v$.

Since these projections are mutually orthogonal, ie. $P_{v} P_{u}=0$ whenever $v \neq u$, the subspaces $P_{v} \mathcal{H}$ are mutually orthogonal subspaces of $\mathcal{H}$. The relation (CK1) implies that the initial space of the partial isometry $S_{e}$ is $P_{s(e)} \mathcal{H}\left(\left[10\right.\right.$, Proposition A.4]) and relation (CK2) informs us that $S_{e} S_{e}^{*}$ is dominated by $P_{r(e)}$, and as such we have that $S_{e} \mathcal{H} \subset P_{r(e)}$ ([10, Proposition A.1]). Thus $S_{e}$ is an isometry of $P_{s(e)}$ onto a closed subspace of $P_{r(e)}$ giving us the relation

$$
S_{e}=P_{s(e)} S_{e}=S_{e} P_{r(e)}
$$

Notice that these relations are strikingly similar to the Leavitt path algebra relations, the main difference being that one may sum infinitely many terms over a $C^{*}$-algebra and the field is strictly $\mathbb{C}$. In fact, one should notice that $\{S, P\}=\left\{S_{e}\right\} \cup\left\{P_{v}\right\} \cup\left\{S_{e}^{*}\right\}$ form a Leavitt E-family inside $B(\mathcal{H})$. Since $S_{e}^{*}$ is the adjoint of $S_{e}$ it is not necessary for $\left\{S_{e}^{*}: e \in E^{1}\right\}$ to be mentioned in the definition of Cuntz-Krieger E-families. 
We denote the $C^{*}$-algebra generated by a Cuntz-Krieger E-family $\{S, P\}$ in $B(\mathcal{H})$ as $C^{*}(S, P)$ and investigate the ${ }^{*}$-algebraic consequences of the Cuntz-Krieger relations.

Proposition 2.3.1. Given a row-finite graph $E$ and a Cuntz-Krieger E-family $\{S, P\}$ in a $C^{*}$-algebra $B$. Then for $e, f \in E^{1}$ and $\mu, \nu \in E^{*}$ :

(i) $S_{e} S_{f} \neq 0 \Longrightarrow s(e)=r(f)$;

(ii) $S_{e}^{*} S_{f}^{*} \neq 0 \Longrightarrow r(e)=s(f)$;

(iii) $S_{e} S_{f}^{*} \neq 0 \Longrightarrow s(e)=s(f)$;

(iv) $\left(S_{e} S_{e}^{*}\right)\left(S_{f} S_{f}^{*}\right)=\left\{\begin{array}{ll}S_{e} S_{e}^{*} & \text { if } e=f \\ 0 & \text { else }\end{array}\right.$ (this implies that $\left\{S_{e} S_{e}^{*}: e \in E^{1}\right\}$ are mutually orthogonal);

(v) $S_{\mu} S_{\nu} \neq 0 \Longrightarrow \mu \nu \in E^{*}$;

(vi) $S_{\mu} S_{\nu}^{*} \neq 0 \Longrightarrow s(\mu)=s(\nu)$;

(vii) $S_{\mu}^{*} S_{\nu}^{*} \neq 0 \Longrightarrow \nu \mu \in E^{*}$;

(viii) if $|\mu|=|\nu|$ and $\mu \neq \nu$, then $\left(S_{\mu} S_{\mu}^{*}\right)\left(S_{\nu} S_{\nu}^{*}\right)=0$;

(ix) $S_{\mu}^{*} S_{\nu}= \begin{cases}S_{\mu^{\prime}}^{*} & \text { if } \mu=\nu \mu^{\prime} \text { for some } \mu^{\prime} \in E^{*} \\ S_{\nu^{\prime}} & \text { if } \nu=\mu \nu^{\prime} \text { for some } \nu^{\prime} \in E^{*} \\ 0 & \text { else. }\end{cases}$

Proof. As $\{S, P\}$ form a Leavitt E-family we can apply the same calculations from Proposition 2.2.2 and Corollary 2.2.3 to see this is true.

In fact the calculations from Proposition 2.2.2 and Corollary 2.2.3 give us the following corollary as per [10, Corollary 1.15]: 
Corollary 2.3.2. Suppose that $E$ is a row-finite directed graph and $\{S, P\}$ is a Cuntz-Krieger E-family in a $C^{*}$-algebra $B$. Then for paths $\mu, \nu, \alpha, \beta \in E^{*}$ we have

$$
\left(S_{\mu} S_{\nu}^{*}\right)\left(S_{\alpha} S_{\beta}^{*}\right)= \begin{cases}\left(S_{\mu \alpha^{\prime}}\right) S_{\beta}^{*} & \text { if } \alpha=\nu \alpha^{\prime} \text { for some } \alpha^{\prime} \in E^{*} \\ S_{\mu}\left(S_{\beta \nu^{\prime}}\right)^{*} & \text { if } \nu=\alpha \nu^{\prime} \text { for some } \nu^{\prime} \in E^{*} \\ 0 & \text { else. }\end{cases}
$$

In particular, it follows that every non-zero finite product of the partial isometries $S_{e}$ and $S_{f}^{*}$ has the form $S_{\mu} S_{\nu}^{*}$ for some $\mu, \nu \in E^{*}$ with $s(\mu)=s(\nu)$.

Proof. This proof follows the proof of Lemma 2.2.4 using the equivalent relations from Proposition 2.3.1.

Corollary 2.3.3. [10, Corollary 1.16] If $\{S, P\}$ is a Cuntz-Krieger E-family in a $C^{*}$-algebra $B$ for a row-finite graph $E$, then

$$
C^{*}(S, P)=\overline{\operatorname{span}}\left\{S_{\mu} S_{\nu}^{*}: \mu, \nu \in E^{*}, s(\mu)=s(\nu)\right\} .
$$

Proof. Corollary 2.3.2 implies that $\operatorname{span}\left\{S_{\mu} S_{\nu}^{*}: \mu, \nu \in E^{*}, s(\mu)=s(\nu)\right\}$ is a sub-algebra of $C^{*}(S, P)$. Since

$$
\left(S_{\mu} S_{\nu}^{*}\right)^{*}=S_{\nu} S_{\mu}^{*} \in \operatorname{span}\left\{S_{\mu} S_{\nu}^{*}: \mu, \nu \in E^{*}, s(\mu)=s(\nu)\right\}
$$

it is a *-sub-algebra; therefore its closure is a $C^{*}$-sub-algebra. Now, $C^{*}(S, P)$ is the smallest $C^{*}$-algebra containing the generators $S_{e}$ and $P_{v}$ and since $S_{e}=S_{e} S_{s(e)}^{*}$ and $P_{v}=S_{v} S_{v}^{*}$ which are both in $\operatorname{span}\left\{S_{\mu} S_{\nu}^{*}: \mu, \nu \in E^{*}, s(\mu)=\right.$ $s(\nu)\}$ we have that

$$
C^{*}(S, P)=\overline{\operatorname{span}}\left\{S_{\mu} S_{\nu}^{*}: \mu, \nu \in E^{*}, s(\mu)=s(\nu)\right\}
$$

Now, [10, Proposition 1.21] states that for any row-finite directed graph $E$, there is a $C^{*}$-algebra $C^{*}(E)$ generated by a universal Cuntz-Krieger E-family $\{s, p\}$ such that for every Cuntz-Krieger E-family $\{T, Q\}$ in a 
$C^{*}$-algebra $B$, there is a homomorphism $\pi_{T, Q}$ of $C^{*}(E)$ into $B$ satisfying $\pi_{T, Q}\left(s_{e}\right)=T_{e}$ for every $e \in E^{1}$ and $\pi_{T, Q}\left(p_{v}\right)=Q_{v}$ for every $v \in E^{0}$. We call $C^{*}(E)$ the graph $C^{*}$-algebra of the graph $E$. In this thesis $\{s, p\}$ will always refer to the universal family which generates $C^{*}(E)$.

Suppose $E$ is a row-finite graph, and $\{s, p\}$ is the universal CuntzKrieger $E$-family. From the discussion above we can see that $\{s, p\}$ form a Leavitt $E$-family, and by the universal property of $L_{K}(E)$ (Remark 2.2.1), there exists a homomorphism $\varphi: L_{\mathbb{C}}(E) \rightarrow C^{*}(E)$ such that $\varphi(v)=p_{v}$, $\varphi(e)=s_{e}$, and $\varphi\left(e^{*}\right)=s_{e}^{*}$. Proving that $\varphi$ is injective directly would be challenging; however, The Graded Uniqueness Theorem [14, Theorem 5.3] states that $\varphi$ is injective since both $L_{\mathbb{C}}(E)$ and $C^{*}(E)$ are graded, which we discuss in the following chapter. This is an example of how the grading in both $L_{\mathbb{C}}(E)$ and $C^{*}(E)$ plays a critical role in informing us about the strong connection between these objects and their structure. From now on we will identify $\varphi\left(L_{\mathbb{C}}(E)\right)$ as $L_{\mathbb{C}}(E)$; in particular, we will write $s_{\mu} s_{\nu}^{*}$ instead of $\mu \nu^{*}$ and $p_{v}$ rather than $v$.

Earlier we said that $L_{\mathbb{C}}(E)=\operatorname{span}\left\{s_{\mu} s_{\nu}^{*}: \mu, \nu \in E^{*}, s(\mu)=s(\nu)\right\}$, so we can see exactly how the Leavitt path algebra $L_{\mathbb{C}}(E)$ sits densely inside $C^{*}(E)=\overline{\operatorname{span}}\left\{s_{\mu} s_{\nu}^{*}: \mu, \nu \in E^{*}, s(\mu)=s(\nu)\right\}$. 


\section{Chapter 3}

\section{Gradings}

We say that a ring $A$ is graded by a group $G$ if $A=\oplus_{g \in G} A_{g}$, where each $A_{g}$ is an additive subgroup of $A$ such that the pointwise product $A_{g} \cdot A_{h} \subseteq A_{g h}$ for every $g, h \in G$. More generally for an algebra $A, A_{g}$ is a subspace of $A$ for every $g \in G$ and the product $A_{g} \cdot A_{h}=\operatorname{span}\left\{a b: a \in A_{g}, b \in A_{h}\right\}$ for every $g, h \in G$. This implies that every element of $A$ can be written as a unique sum of elements from the $A_{g}$ 's. We refer to these subgroups as the grading of $A$, or grading subspaces whenever $A$ is an algebra.

A $C^{*}$-algebra is graded if it contains a dense graded sub-ring; however, unlike in the algebraic sense, we do not necessarily have a canonical way to write an element of a graded $C^{*}$-algebra in terms of elements from its grading.

In this chapter we begin by showing that $L_{\mathbb{C}}(E)$ is $\mathbb{Z}$-graded. We then prove the graph $C^{*}$-algebras of row-finite graphs are also $\mathbb{Z}$-graded, and that the grading subspaces of $C^{*}(E)$ are the closed spans of the grading subspaces of the Leavitt path algebra sitting densely inside it. We then discuss the structure of the grading subspace associated with 0 for both Leavitt path algebras and graph $\mathrm{C}^{*}$-algebras. Both are isomorphic to the direct limit of matrix algebras - a fact we use in the argument for the main result of this thesis. The grading subspaces of the graph $\mathrm{C}^{*}$-algebra col- 
lectively form an analytic structure known as a Fell bundle, which we describe more thoroughly towards the end of this chapter. We also introduce the notion of saturated Fell bundles, which are a class of Fell bundles satisfying a condition similar to the description of strong grading in the algebraic sense.

\subsection{The $\mathbb{Z}$-grading of $L_{\mathbb{C}}(E)$}

In Chapter 2 we saw that $L_{\mathbb{C}}(E)=\operatorname{span}\left\{s_{\mu} s_{\nu}^{*}: \mu, \nu \in E^{*}, s(\mu)=s(\nu)\right\}$. In [1, Corollary 2.1.5] Abrams states that for any graph $E$ the Leavitt path algebra $L_{K}(E)$ is $\mathbb{Z}$-graded with grading subspaces

$$
L_{\mathbb{C}}(E)_{n}:=\operatorname{span}\left\{s_{\mu} s_{\nu}^{*}: \mu, \nu \in E^{*},|\mu|-|\nu|=n, s(\mu)=s(\nu)\right\} .
$$

In this section we verify this for row-finite graphs without sources using an adaptation of [11, Proposition C.1] for Leavitt path algebras. It is difficult to show that the $L_{\mathbb{C}}(E)_{n}$ are linearly independent due to the multiple ways we can represent elements of a Leavitt path algebra. In order to do this we use tensor products between the Leavitt path algebra $L_{\mathbb{C}}(E)$ and the group algebra $\mathbb{C} \mathbb{Z}$. For more information about group algebras see [6, pg. 227]. We now define and review some basic properties of the tensor products. The following discussion and proposition is a summary of $[7$, Chapter 8, pg. 319-321] adapted to the specific setting of vector spaces.

For any two vector spaces $V$ and $W$ over a field $K$ we simultaneously construct a vector space $V \otimes W$ and a bilinear $\operatorname{map} \varphi: V \times W \rightarrow V \otimes W$. We begin by constructing a free vector space $F$ generated by $V \times W$. We then consider the subspace of $S$ of $F$ spanned by elements

$$
\begin{aligned}
& \left(k_{1} v_{1}+k_{2} v_{2}, w\right)-k_{1}\left(v_{1}, w\right)+k_{2}\left(v_{2}, w\right), \text { and } \\
& \left(v, k_{1} w_{1}+k_{2} w_{2}\right)-k_{1}\left(v, w_{1}\right)+k_{2}\left(v, w_{2}\right)
\end{aligned}
$$

for $k_{1}, k_{2} \in K, v, v_{1}, v_{2} \in V$ and $w, w_{1}, w_{2} \in W$, and write $V \otimes W$ for the quotient space $F / S$, and call this the tensor product of $V$ and $W$. 
Then $V \otimes W$ is a vectors space generated by vectors of the form $v \otimes w$ for vectors $v \in V$, and $w \in W$. The relation $\otimes: V \times W \rightarrow V \otimes W$ is such that for every $v, v_{1}, v_{2} \in V, w, w_{1}, w_{2} \in W$ and $c \in K$ we have:

(i) $c(v \otimes w)=(c v) \otimes w=v \otimes(c w)$

(ii) $\left(v_{1}+v_{2}\right) \otimes w=v_{1} \otimes w+v_{2} \otimes w$

(iii) $v \otimes\left(w_{1}+w_{2}\right)=v \otimes w_{1}+v \otimes w_{2}$.

And thus $\varphi:(v, w) \mapsto v \otimes w$ is bilinear.

Proposition 3.1.1. (Universal Property of Tensor Product Vector Spaces) The vector spaces $V, W$, and the associated bilinear map $\varphi: V \times W \rightarrow V \otimes W$ have the property that for any bilinear map $b: V \times W \rightarrow X$ where $X$ is some vector space, there exists a linear map $b^{\prime}: V \otimes W \rightarrow X$ such that $b(v, w)=b^{\prime} \circ \varphi(v, w)=$ $b^{\prime}(v \otimes w)$ for any $v \in V$ and $w \in W$. Thus $L(V \otimes W, X)=B(V \times W, X)$.

The following Lemma can be found in [13, Lemma B.1]:

Lemma 3.1.2. If $A$ and $B$ are *algebras over a field $K$, then there is a unique algebra structure on the vector space tensor product over $A \otimes B$ such that

$$
(a \otimes b)(c \otimes d)=a c \otimes b d \quad \text { and } \quad(a \otimes b)^{*}=a^{*} \otimes b^{*}
$$

for every $a, c \in A$ and $b, d \in B$.

Proof. We verify the first relation, for the proof of the second relation see [13, Lemma B.1]. Fix $a \in A$ and $b \in B$. Then the map $(c, d) \mapsto a c \otimes b d$ is bilinear for all $c \in A$ and $d \in B$ by the properties of elements of $A \otimes B$, and multiplication in $A$ and $B$. So, by the Universal Property of Tensor Products of Vector Spaces there exists a linear map $L_{a, b}: c \otimes d \rightarrow a c \otimes b d$.

Let us consider the map $L$ whereby $L(a, b) \mapsto L_{a} \otimes L_{b}=L_{a, b}$ where $L_{a}$ and $L_{b}$ are linear mappings with respect to $a$ and $b$. Clearly, this is a bilinear mapping from $A \times B$ to $L(A \otimes B)$ which is the space of linear mappings of $A \times B$, so there exists a linear map $\bar{L}: A \otimes B \rightarrow L(A \otimes B)$ such that 
$\bar{L}(a \otimes b)=L_{a, b}$. So the required product can be defined as $(a \otimes b)(c \otimes d)=$ $\bar{L}(a \otimes b)(c \otimes d)$.

We can check that the required laws for associativity and distributivity for multiplication over an algebra hold. First we check to see if the multiplication as defined is associative:

$$
\begin{aligned}
((a \otimes b)(c \otimes d))(e \otimes f) & =(\bar{L}(a \otimes b)(c \otimes d))(e \otimes f) \\
& =\left(L_{a, b}(c \otimes d)\right)(e \otimes f) \\
& =(a c \otimes b d)(e \otimes f) \\
& =L_{a c, b d}(e \otimes f) \\
& =a c e \otimes b d f \\
& =L_{a, b}(c e \otimes d f) \\
& =(a \otimes b)(c e \otimes d f) \\
& =(a \otimes b)\left(L_{c, d}(e \otimes f)\right) \\
& =(a \otimes b)((c \otimes d)(e \otimes f))
\end{aligned}
$$

Now we check the distributive properties hold:

$$
\begin{aligned}
(a \otimes b)((c \otimes d)+(e \otimes f)) & =L_{a, b}((c \otimes d)+(e \otimes f)) \\
& =L_{a, b}(c \otimes d)+L_{a, b}(e \otimes f) \\
& =(a \otimes b)(c \otimes d)+(a \otimes b)(e \otimes f), \\
((a \otimes b)+(c \otimes d))(e \otimes f) & =\bar{L}((a \otimes b)+(c \otimes d))(e \otimes f) \\
& =(\bar{L}(a \otimes b)+\bar{L}(c \otimes d))(e \otimes f) \\
& =\left(L_{a, b}+L_{c, d}\right)(e \otimes f) \\
& =L_{a, b}(e \otimes f)+L_{c, d}(e \otimes f) \\
& =(a \otimes b)(e \otimes f)+(c \otimes d)(e \otimes f),
\end{aligned}
$$




$$
\begin{aligned}
(z(a \otimes b))(c \otimes d) & =\bar{L}(z(a \otimes b))(c \otimes d) \\
& =z \bar{L}(a \otimes b)(c \otimes d) \\
& =z((a \otimes b)(c \otimes d)) \\
& =z L_{a, b}(c \otimes d) \\
& =L_{a, b}(z(c \otimes d)) \\
& =(a \otimes b)(z(c \otimes d))
\end{aligned}
$$

verifying the first relation.

Now we are able to verify that for a row-finite graph without sources, $L_{\mathbb{C}}(E)$ is $\mathbb{Z}$-graded. To do this we adapt the proof of [11, Proposition Proposition C.1] to the context of Leavitt path algebras.

Proposition 3.1.3. Let $E$ be a directed row-finite graph with no sources. Then $L_{\mathbb{C}}(E)$ is a $\mathbb{Z}$-graded algebra so that

$$
L_{\mathbb{C}}(E)=\bigoplus_{n \in \mathbb{Z}} L_{\mathbb{C}}(E)_{n} .
$$

Proof. Define

$$
L_{\mathbb{C}}(E)_{n}:=\operatorname{span}\left\{s_{\mu} s_{\nu}^{*}:|\mu|-|\nu|=n\right\} .
$$

Clearly, the subspaces $L_{\mathbb{C}}(E)_{n}$ span $L_{\mathbb{C}}(E)$, so we must check that

$$
L_{\mathbb{C}}(E)_{n} \cap L_{\mathbb{C}}(E)_{m}=\{0\} \quad \forall m, n \in \mathbb{Z} \text { with } n \neq m .
$$

To see this is true we show that these subspaces are linearly independent using an argument from coaction theory. Consider the group algebra of the integers, $\mathbb{C Z}$. First, we take $\eta: E^{*} \rightarrow \mathbb{Z}$ such that $\mu \mapsto|\mu|$ for $\mu \in E^{*}$. Define $\tau: \mathbb{C Z} \rightarrow \mathbb{C}$ by $\tau(y)=y_{0}$ where $y=\sum_{n \in \mathbb{Z}} y_{n} \delta_{n} \in \mathbb{C} \mathbb{Z}$. For arbitrary 
$k \in \mathbb{C}$ and $y, x \in \mathbb{C} \mathbb{Z}:$

$$
\begin{aligned}
\tau(k y+x) & =\tau\left(\sum_{n \in \mathbb{Z}} k y_{n} \delta_{n}+\sum_{n \in \mathbb{Z}} x_{n} \delta_{n}\right) \\
& =\tau\left(\sum_{n \in \mathbb{Z}}\left(k y_{n}+x_{n}\right) \delta_{n}\right) \\
& =k y_{0}+x_{0} \\
& =k \tau(y)+\tau(x) .
\end{aligned}
$$

So $\tau$ is a linear transformation. For each $g \in \mathbb{Z}$ we define $\tau_{g}(y):=\tau\left(\delta_{g}^{-1} y\right)=$ $y_{g}$. We claim that given the Cuntz-Krieger $E$-family $\{s, p\}$, the elements $s_{\mu} \otimes \eta(\mu)$ generate a Leavitt E-family in $L_{\mathbb{C}}(E) \otimes \mathbb{C Z}$ by checking they agree with the Leavitt path algebra relations. Fix $p_{v}, p_{w} \in L_{\mathbb{C}}(E)$, then

$$
\begin{aligned}
\left(p_{v} \otimes \eta(v)\right)\left(p_{w} \otimes \eta(w)\right) & =\left(p_{v} p_{w} \otimes \eta(v w)\right) \\
& = \begin{cases}p_{v} \otimes \eta(v) & \text { if } v=w \\
0 & \text { else. }\end{cases}
\end{aligned}
$$

So the first relation is satisfied. For $s_{e} \in L_{\mathbb{C}}(E)$ we have

$$
r(e) s_{e} \otimes \eta(r(e) e)=s_{e} \otimes \eta(e)=s_{e} s(e) \otimes \eta(e s(e))
$$

satisfying the second relation. We can see the third relation is satisfied using a similar calculation.

To see that (CK1) holds fix $s_{e}, s_{f} \in L_{\mathbb{C}}(E)$, then

$$
\begin{aligned}
\left(s_{e} \otimes \eta(e)\right)^{*}\left(s_{e} \otimes \eta(e)\right) & =s_{e}^{*} s_{e} \otimes \eta\left(e^{*} e\right) \\
& =p_{s(e)} \otimes 0 \\
& =p_{s(e)} \otimes \eta(v) .
\end{aligned}
$$


Lastly to see that (CK2) is satisfied fix $p_{v} \in L_{\mathbb{C}}(E)$, then

$$
\begin{aligned}
p_{v} \otimes \eta(v) & =\left(\sum_{e \in E^{*}: r(e)=v}\left(s_{e} s_{e}^{*}\right)\right) \otimes 0 \\
& =\sum_{e \in E^{*}: r(e)=v}\left(\left(s_{e} s_{e}^{*}\right) \otimes 0\right) \\
& =\sum_{e \in E^{*}: r(e)=v}\left(s_{e} s_{e}^{*}\right) \otimes \eta\left(e e^{*}\right) \\
& =\sum_{e \in E^{*}: r(e)=v}\left(s_{e} \otimes \eta(e)\right)\left(s_{e} \otimes \eta(e)\right)^{*} .
\end{aligned}
$$

Hence, by the universal property of Leavitt path algebras, Remark 2.2.1, there is a homomorphism $\pi_{s \otimes \eta}: L_{\mathbb{C}}(E) \rightarrow L_{\mathbb{C}}(E) \otimes \mathbb{C Z}$ such that $\pi_{s \otimes \eta}\left(s_{\mu}\right)=$ $s_{\mu} \otimes \eta(\mu)$. The map $(x \otimes c) \mapsto x \tau_{g}(c)$ is bilinear by the scalar multiplication of tensor products, so by the universal property of tensor products of vector spaces, there is a well-defined linear map $\iota \otimes \tau_{g}$ from $L_{\mathbb{C}}(E) \otimes \mathbb{C}$ to $L_{\mathbb{C}}(E)$ where $\iota$ is the identity map on $L_{\mathbb{C}}(E)$, such that $\iota \otimes \tau_{g}(x \otimes h)=x \tau_{g}(h)$. We can see that

$$
\begin{aligned}
\left(\iota \otimes \tau_{g}\right) \circ \pi_{s \otimes \eta}\left(s_{\mu} s_{\nu}^{*}\right) & =\left(\iota \otimes \tau_{g}\right)\left(s_{\mu} s_{\nu}^{*} \otimes \eta\left(\mu \nu^{*}\right)\right) \\
& = \begin{cases}s_{\mu} s_{\nu}^{*} & \text { if }|\mu|-|\nu|=g \\
0 & \text { else. }\end{cases}
\end{aligned}
$$

To see linear independence take the finite sum $a=\sum_{n \in F} a_{n}$ where each 
$a_{n} \in L_{\mathbb{C}}(E)_{n}$ and $a=0$ in $L_{\mathbb{C}}(E)$. For all $g \in \mathbb{Z}$ we have

$$
\begin{aligned}
0 & =\left(i d \otimes \tau_{g}\right) \circ \pi_{s \otimes \eta}\left(\sum_{n \in F} a_{n}\right) \\
& =\left(i d \otimes \tau_{g}\right) \circ\left(\sum_{n \in F} \pi_{s \otimes \eta}\left(a_{n}\right)\right) \\
& =\left(i d \otimes \tau_{g}\right)\left(\sum_{n \in F}\left(a_{n} \otimes n\right)\right) \\
& =\sum_{n \in F}\left(i d \otimes \tau_{g}\right)\left(a_{n} \otimes n\right) \\
& =\sum_{n \in F} a_{n} \tau_{g}(n) \\
& =a_{g} .
\end{aligned}
$$

Therefore the subspaces $L_{\mathbb{C}}(E)_{n}$ are linearly independent, and thus their direct sum is equal to $L_{\mathbb{C}}(E)$.

It remains to show that $L_{\mathbb{C}}(E)_{n} L_{\mathbb{C}}(E)_{m} \subseteq L_{\mathbb{C}}(E)_{n+m}$. To see this, fix $a \in$ $L_{\mathbb{C}}(E)_{n}$ and $b \in L_{\mathbb{C}}(E)_{m}$, then $a b$ can be written as a linear combination of elements of the form $\left(s_{\mu} s_{\nu}^{*}\right)\left(s_{\alpha} s_{\beta}^{*}\right)$ for $\mu, \nu, \alpha, \beta \in E^{*}$ such that $|\mu|-|\nu|=n$, and $|\alpha|-|\beta|=m$. From Lemma 2.2.4 we know that

$$
\left(s_{\mu} s_{\nu}^{*}\right)\left(s_{\alpha} s_{\beta}^{*}\right)= \begin{cases}s_{\mu \alpha^{\prime}} s_{\beta}^{*} & \text { if } \alpha=\nu \alpha^{\prime} \text { for some } \alpha^{\prime} \in E^{*} \\ s_{\mu} s_{\beta \nu^{\prime}}^{*} & \text { if } \nu=\alpha \nu^{\prime} \text { for some } \nu^{\prime} \in E^{*} \\ 0 & \text { else. }\end{cases}
$$

Clearly, $0 \in L_{\mathbb{C}}(E)_{n+m}$, so all we need to do is check the other two cases.

Suppose $\left(s_{\mu} s_{\nu}^{*}\right)\left(s_{\alpha} s_{\beta}^{*}\right)=s_{\mu \alpha^{\prime}} s_{\beta}^{*}$, for some $\alpha^{\prime} \in E^{*}$, then $\left|\alpha^{\prime}\right|=|\alpha|-|\nu|$. Then

$$
\begin{aligned}
\left|\mu \alpha^{\prime}\right|-|\beta| & =|\mu|+\left|\alpha^{\prime}\right|-|\beta| \\
& =|\mu|+|\alpha|-|\nu|-|\beta| \\
& =n+m .
\end{aligned}
$$

Similarly, we see that if $\left(s_{\mu} s_{\nu}^{*}\right)\left(s_{\alpha} s_{\beta}^{*}\right)=s_{\mu} s_{\beta \nu^{\prime}}^{*}$ for some $\nu^{\prime} \in E^{*}$ then $|\mu|-$ $\left|\beta \nu^{\prime}\right|=n+m$. Thus $\left(s_{\mu} s_{\nu}^{*}\right)\left(s_{\alpha} s_{\beta}^{*}\right)=a b \in L_{\mathbb{C}}(E)_{n+m}$. 


\subsection{The $\mathbb{Z}$-grading of Graph $C^{*}$-algebras}

The following definition for graded $C^{*}$-algebras can be found in [5, p. 125]. Let $A$ be a $C^{*}$-algebra and $G$ be a group. We say that a linearly independent collection $\left\{A_{\sigma}\right\}_{\sigma \in G}$ of closed subspaces of $A$ is a $C^{*}$-grading for $A$, if $\bigoplus_{\sigma \in G} A_{\sigma}$ is dense in $A$, and for every $\sigma$ and $\tau$ in $G$ one has that

(i) $A_{\sigma} \cdot A_{\tau} \subseteq A_{\sigma \tau}$

(ii) $A_{\sigma}^{*} \subseteq A_{\sigma^{-1}}$.

If $A$ has a $\mathrm{C}^{*}$-grading we say that $A$ is $\mathrm{C}^{*}$-graded; moreover, if it is clear that $A$ is a $C^{*}$-algebra we simply say that $A$ is graded.

In this section we show that $C^{*}(E)$ is $\mathbb{Z}$-graded with grading subspaces

$$
C^{*}(E)_{n}=\overline{L_{\mathbb{C}}(E)_{n}}:=\overline{\operatorname{span}}\left\{s_{\mu} s_{\nu}^{*}: s_{\mu} s_{\nu}^{*} \in L_{\mathbb{C}}(E)_{n}\right\} .
$$

In order to do this we need to show that $\left\{\overline{L_{\mathbb{C}}(E)_{n}}\right\}_{n \in \mathbb{Z}}$ is a collection of linearly independent subspaces which are dense in $C^{*}(E)$. Showing that the subspaces $\left\{\overline{L_{\mathbb{C}}(E)_{n}}\right\}_{n \in \mathbb{Z}}$ are linearly independent is tricky in an analytic setting because we need to show that the closures of the $L_{\mathbb{C}}(E)_{n}$ do not intersect. To do this we use a faithful map $\Phi_{n}$ to project $C^{*}(E)$ onto $\overline{L_{\mathbb{C}}(E)_{n}}$ for all $n \in \mathbb{Z}$, which averages $C^{*}(E)$ over the gauge action $\gamma$ of the unit circle $\mathbb{T}$ on $C^{*}(E)$.

In order to construct this map we establish the existence of the gauge action of $\mathbb{T}$ on $C^{*}(E)$. The following Proposition follows [10, Proposition 2.1].

Theorem 3.2.1. Let $E$ be a row-finite directed graph. Then there is a topological group action $\gamma$ of $\mathbb{T}$ on $C^{*}(E)$ such that $\gamma_{z}\left(s_{e}\right)=z s_{e}$ for every $e \in E^{1}$ and $\gamma_{z} p_{v}=p_{v}$ for every $v \in E^{0}$. 
Proof. We take $C^{*}(E)$ generated by the Cuntz-Krieger $E$-family $\{s, p\}=$ $\left\{s_{e}, p_{v}\right\}$, and fix $z \in \mathbb{T}$. Then $|z|=1$ and $\{z s, p\}=\left\{z s_{e}, p_{v}\right\}$ is a CuntzKrieger $E$-family since

$$
\left(z s_{e}\right)^{*}\left(z s_{e}\right)=|z| s_{e}^{*} s_{e}=p_{s(e)}
$$

and

$$
\sum_{e \in E^{1} ; r(e)=v}\left(z s_{e}\right)\left(z s_{e}\right)^{*}=\sum_{e \in E^{1} ; r(e)=v}|z| s_{e} s_{e}^{*}=p_{v} ;
$$

satisfying the Cuntz-Krieger relations. Since

$$
C^{*}(s, p)=C^{*}(E)=\overline{\operatorname{span}}\left\{s_{\mu} s_{\nu}^{*}: \mu, \nu \in E^{*}\right\},
$$

$C^{*}(z s, p)$ is a sub-algebra of $C^{*}(E)$ because $z s_{e}$ is a scalar multiple of $s_{e}$. Likewise, $s_{e}=\bar{z} z s_{e}$ so $C^{*}(E)$ is contained in $C^{*}(z s, p)$, therefore $\{z s, p\}$ generates $C^{*}(E)$.

We can also see that if $\{T, Q\}$ is a Cuntz-Krieger $E$-family in a $C^{*}(E)$ algebra $B$, then $\{\bar{z} T, Q\}$ is a Cuntz-Krieger E-family in $B$. Using the homomorphism $\pi_{\bar{z} T, Q}$ of $C^{*}(E)$ into $B$ as described in [10, Proposition 1.21] we have:

$$
\begin{aligned}
\pi_{\bar{z} T, Q}\left(z s_{e}\right) & =z \pi_{\bar{z} T, Q}\left(s_{e}\right) \\
& =z\left(\bar{z} T_{e}\right) \\
& =|z| T_{e} \\
& =T_{e} .
\end{aligned}
$$

With the homomorphism $\rho_{T, Q}:=\pi_{\bar{z} T, Q}$, the pair $\left(C^{*}(E),\{z s, p\}\right)$ has the property described in [10, Corollary 1.22] so there is an isomorphism $\gamma_{z}$ of $C^{*}(E)$ onto $C^{*}(E)$ such that $\gamma_{z}\left(s_{e}\right)=z s_{e}$ for every $e \in E^{1}$ and $\gamma_{z} p_{v}=p_{v}$ for every $v \in E^{0}$.

To see that $\gamma$ is be a continuous group action, we must first show that it is a group action. Fix $w \in \mathbb{T}$, then 


$$
\begin{aligned}
\gamma_{z} \circ \gamma_{w}\left(s_{e}\right) & =\gamma_{z}\left(\gamma_{w}\left(s_{e}\right)\right) \\
& =\gamma_{z}\left(w s_{e}\right) \\
& =z w s_{e} \\
& =\gamma_{z w}\left(s_{e}\right) .
\end{aligned}
$$

In addition, we can see that when $z=1, \gamma_{z}$ acts as the identity over $C^{*}(E)$ using a straightforward calculation. So $\gamma$ is an action of $\mathbb{T}$ on $C^{*}(E)$, and $\gamma$ is a homomorphism of $\mathbb{T}$ into Aut $C^{*}(E)$ by [6, Theorem 4.5].

To show $\gamma$ is continuous under multiplication, we use an $\varepsilon / 3$ argument. Fix $z \in \mathbb{T}, a \in C^{*}(E)$ and $\varepsilon>0$. Choose $c:=\sum_{\mu, \nu \in E^{*}} \lambda_{\mu, \nu} s_{\mu} s_{\nu}^{*}$ such that $\|a-c\|<\varepsilon / 3$. Notice that $\gamma_{z}\left(s_{\mu}\right)=z^{|\mu|} s_{\mu}$, and $\gamma_{z}\left(s_{\mu}^{*}\right)=\left(z^{|\mu|} s_{\mu}\right)^{*}=z^{-|\mu|} s_{\mu}^{*}$ because $|\lambda|=1$. Hence $\gamma_{z}\left(s_{\mu} s_{\nu}^{*}\right)=z^{|\mu|-|\nu|} s_{\mu} s_{\nu}^{*}$. By the continuity of scalar multiplication

$$
w \longmapsto \sum_{\mu, \nu \in E^{*}} \lambda_{\mu, \nu} w^{|\mu|-|\nu|} s_{\mu} s_{\nu}^{*}
$$

is continuous so there exists some $\delta>0$ such that

$$
|w-z|<\delta \Longrightarrow\left\|\gamma_{w}(c)-\gamma_{z}(c)\right\|<\varepsilon / 3 .
$$

We also know that automorphisms of $\mathrm{C}^{*}$-algebras preserve the norm so that $\left\|\gamma_{z}(a-c)\right\|<\varepsilon / 3$ for every $z \in \mathbb{T}$. Thus, for any $w \in \mathbb{T}$ such that $|w-z|<\delta$ we have the following inequality:

$$
\begin{aligned}
\left\|\gamma_{w}(a)-\gamma_{z}(a)\right\| & \leq\left\|\gamma_{w}(a-c)\right\|+\left\|\gamma_{w}(c)-\gamma_{z}(a)\right\| \\
& \leq\left\|\gamma_{w}(a-c)\right\|+\left\|\gamma_{w}(c)-\gamma_{z}(c)\right\|+\left\|\gamma_{z}(c)-\gamma_{z}(a)\right\| \\
& <3(\varepsilon / 3)=\varepsilon
\end{aligned}
$$

as required.

Now that we have defined the gauge action we can use it to project $C^{*}(E)$ onto the subspaces $\overline{L_{\mathbb{C}}(E)_{n}}$ for every $n \in \mathbb{Z}$. Our method for doing 
this is motivated by the map which projects $C^{*}(E)$ onto the fixed point algebra

$$
C^{*}(E)^{\gamma}:=\left\{a \in C^{*}(E): \gamma_{z}(a)=a, \forall z \in \mathbb{T}\right\} .
$$

We show that $C^{*}(E)^{\gamma}$ is the same as $\overline{L_{\mathbb{C}}(E)_{0}}$. We use the following lemmas which can be found in [10, Chapter 3] to construct a faithful map $\Phi: C^{*}(E) \rightarrow C^{*}(E)^{\gamma}$ called a conditional expectation.

Lemma 3.2.2. Suppose that $A$ is a $C^{*}$-algebra and $f: \mathbb{T} \longrightarrow A$ is a continuous function. Then there is a unique element $\int_{\mathbb{T}} f(z) d z$ of $A$ such that, for every representation $\pi$ of $A$ on $\mathcal{H}$ and $h, k \in \mathcal{H}$ we have

$$
\left(\pi\left(\int_{\mathbb{T}} f(z) d z\right) h \mid k\right)=\int_{\mathbb{T}}(\pi(f(z)) h \mid k) d z .
$$

Which gives us:

(i) $b\left(\int_{\mathbb{T}} f(z) d z\right)=\int_{\mathbb{T}} b f(z) d z$ for $b \in A$

(ii) $\left\|\int_{\mathbb{T}} f(z) d z\right\| \leq \int_{\mathbb{T}}\|f(z)\| d z$

(iii) $\phi\left(\int_{\mathbb{T}} f(z) d z\right)=\int_{\mathbb{T}} \phi(f(z)) d z$ for every homomorphism $\phi: A \rightarrow B$

(iv) for $w \in \mathbb{T}, \int_{\mathbb{T}} f(w z) d z=\int_{\mathbb{T}} f(z) d z$.

Proof. We follow the proof given in [10, Lemma 3.1], with additional information from [13, pg.275]. We choose a faithful representation $\rho: A \rightarrow$ $B(\mathcal{H})$. The map $(h, k) \mapsto \int_{\mathbb{T}}(\rho(f(z)) h \mid k) d z$ is sesquilinear given the linearity of integration and sesquilinearity of the inner product, thus there exists a linear operator $T \in B(\mathcal{H})$ satisfying $(T h \mid k)=\int_{\mathbb{T}}(\rho(f(z)) h \mid k) d z$ for all $h, k \in \mathcal{H}$ by [9, Theorem 2.3.6]. We can see that $T$ is bounded by $\|f\|_{1}$ since

$$
\begin{aligned}
(T h \mid k) & =\int_{\mathbb{T}}(\rho(f(z)) h \mid k) d z \\
& \leq\|f\|_{1}\|h\|\|k\| \text { for all } h, k \in \mathcal{H} .
\end{aligned}
$$


Now we want to show that $T \in \rho(A)$ so that we can define

$$
\int_{\mathbb{T}} f(z) d z:=\rho^{-1}(T) .
$$

To see this we fix $\varepsilon<0$. The Heine-Cantor theorem informs us that $f$ is uniformly continuous since the function $f$ is continuous and $\mathbb{T}$ is compact, so there exists some $\delta$ such that for every $y, z \in \mathbb{T}$,

$$
|y-z|<\delta \text { implies that }\|f(y)-f(z)\|<\varepsilon .
$$

Let $V$ be the open set $B(1, \delta) \cap \mathbb{T}$. If $y^{-1} z \in V$, then

$$
\begin{aligned}
\left|1-y^{-1} z\right|<\delta & \Longrightarrow|y-z|<\delta \\
& \Longrightarrow\|f(y)-f(z)\|<\varepsilon .
\end{aligned}
$$

Now $\{y V\}_{y \in \mathbb{T}}$ is an infinite open cover of $\mathbb{T}$, which is compact, so there are finitely many $y_{i}$ so that $\bigcup_{i} y_{i} V$ is a finite open cover of $\mathbb{T}$. We can make a partition of unity $\left\{\phi_{i}\right\}$ on $\mathbb{T}$ subordinate to $\bigcup_{i} y_{i} V$ in accordance with [13, Lemma 4.34]. The functions $\phi_{i} \in C(\mathbb{T})$ are such that $0 \leq \phi_{i} \leq 1$, and $\operatorname{supp}\left(\phi_{i}\right) \subset\left(y_{i} \cdot V\right)$ for each $i$, satisfying $\sum_{i} \phi_{i}(z)=1$ for all $z \in \mathbb{T}$.

Now

$$
\begin{aligned}
\left\|f-\sum_{i} f\left(y_{i}\right) \phi_{i}\right\|_{1} & =\int_{\mathbb{T}}\left\|f(z)-\sum_{i} f\left(y_{i}\right) \phi_{i}(z)\right\| d z \\
& =\int_{\mathbb{T}}\left\|f(z) \sum_{i} \phi_{i}(z)-\sum_{i} f\left(y_{i}\right) \phi_{i}(z)\right\| d z \\
& =\int_{\mathbb{T}}\left\|\sum_{i}\left(f(z)-f\left(y_{i}\right)\right) \phi_{i}(z)\right\| d z \\
& \leq \int_{\mathbb{T}} \sum_{i}\left\|\left(f(z)-f\left(y_{i}\right)\right) \phi_{i}(z)\right\| d z \\
& \leq \int_{\mathbb{T}} \sum_{i}\left\|f(z)-f\left(y_{i}\right)\right\| \phi_{i}(z) d z \\
& <\int_{\mathbb{T}} \varepsilon \sum_{i} \phi_{i}(z) d z \\
& =\varepsilon .
\end{aligned}
$$


So $\sum_{i} f\left(y_{i}\right) \phi_{i} \rightarrow f$ as $\varepsilon \rightarrow 0$. This implies that

$$
\begin{aligned}
(T h \mid k) & -\left(\sum_{i}\left(\int_{\mathbb{T}} \phi_{i}(z) d z\right) \rho\left(f\left(y_{i}\right)\right) h \mid k\right) \\
& =\int_{\mathbb{T}}(\rho(f(z)) h \mid k) d z-\int_{\mathbb{T}}\left(\left(\sum_{i} \phi_{i}(z) \rho\left(f\left(y_{i}\right)\right)\right) h \mid k\right) d z \\
& =\int_{\mathbb{T}}\left(\left(\rho(f(z))-\sum_{i} \phi_{i}(z) \rho\left(f\left(y_{i}\right)\right)\right) h \mid k\right) d z \\
& \leq\left\|f-\sum_{i} f\left(y_{i}\right) \phi_{i}\right\|_{1}\|h\|\|k\| \\
& <\varepsilon\|h\|\|k\| .
\end{aligned}
$$

So $\left\|T-\sum_{i} \phi_{i}(z) \rho\left(f\left(y_{i}\right)\right)\right\|_{o p} \rightarrow 0$ as $\varepsilon \rightarrow 0$.

Let $c_{i}=\int_{\mathbb{T}} \phi_{i}(z) d z$. Then the sum $\sum_{i} c_{i} \rho\left(f\left(y_{i}\right)\right) \in \rho(A)$ and $\sum_{i} c_{i} \rho\left(f\left(y_{i}\right)\right) \rightarrow \int_{\mathbb{T}} \rho(f(z)) d z=T$. Thus $T \in \rho(A)$. We can define $\int_{\mathbb{T}} f(z) d z:=$ $\rho^{-1}(T)$. Since $\rho$ is isometric, $\left\|\int_{\mathbb{T}} f(z) d z-\sum_{i} c_{i} f\left(y_{i}\right)\right\|<\varepsilon$ which shows that $\int_{\mathbb{T}} f(z) d z$ is unique.

Given any representation $\pi$ of A:

$$
\begin{aligned}
\mid\left(\pi\left(\int_{\mathbb{T}} f(z) d z\right) h \mid k\right) & -\left(\left(\int_{\mathbb{T}} \pi(f(z)) d z\right) h \mid k\right) \mid \\
\leq & \left|\left(\pi\left(\int_{\mathbb{T}} f(z) d z-\sum_{i} c_{i} f\left(y_{i}\right)\right) h \mid k\right)\right| \\
& +\mid\left(\left(\sum_{i} c_{i} \pi\left(f\left(y_{i}\right)-\int_{\mathbb{T}} \pi(f(z)) d z\right) h \mid k\right) \mid\right. \\
& \leq(\varepsilon+\varepsilon)\|h\|\|k\| \\
& =2 \varepsilon\|h\|\|k\|,
\end{aligned}
$$

this proves Equation (3.1) since $\varepsilon$ is arbitrary.

We can use Equation (3.1) to show $(i)$ and (ii) by fixing $b \in A$ and a 
faithful representation $\rho$ as follows:

$$
\begin{aligned}
\left(\rho\left(\int_{\mathbb{T}} b f(z) d z\right) h \mid k\right) & =\int_{\mathbb{T}}(\rho(b f(z)) h \mid k) d z \\
& =\int_{\mathbb{T}}(\rho(b) \rho(f(z)) h \mid k) d z \\
& =\left(\rho(b) \rho\left(\int_{\mathbb{T}} f(z) d z\right) h \mid k\right) \\
& =\left(\rho\left(b \int_{\mathbb{T}} f(z) d z\right) h \mid k\right)
\end{aligned}
$$

and

$$
\begin{aligned}
\left\|\int_{\mathbb{T}} f(z) d z\right\| & =\left\|\rho\left(\int_{\mathbb{T}} f(z) d z\right)\right\|_{o p} \\
& =\left\|\int_{\mathbb{T}} \rho(f(z)) d z\right\|_{o p} \\
& \leq \int_{\mathbb{T}}\|\rho(f(z)) d z\|_{o p} \\
& =\int_{\mathbb{T}}\|f(z) d z\| .
\end{aligned}
$$

For (iii) let $\rho$ be a faithful representation of $B$ and let $\pi=\rho \circ \phi$. Then

$$
\begin{aligned}
\phi\left(\int_{\mathbb{T}} f(z) d z\right) & =\rho^{-1} \circ \pi\left(\int_{\mathbb{T}} f(z) d z\right) \\
& =\rho^{-1}\left(\int_{\mathbb{T}} \pi(f(z)) d z\right) \\
& =\int_{\mathbb{T}} \rho^{-1} \circ \pi(f(z)) d z \\
& =\int_{\mathbb{T}} \phi(f(z)) d z .
\end{aligned}
$$

To show $(i v)$, recall that for a continuous function $g: \mathbb{T} \rightarrow \mathbb{C}$ we write

$$
\int_{\mathbb{T}} f(z) d z=\int_{0}^{1} f\left(e^{2 \pi i t}\right) d t .
$$

We can obtain integrals of continuous functions $g: \mathbb{T} \rightarrow \mathbb{C}$ by using the right hand side of Equation (3.1) which integrates an inner product over 
$\mathbb{T}$. Fix $w \in \mathbb{T}$ with $w=e^{2 \pi i \theta}$, then

$$
\int_{\mathbb{T}} g(w z) d z=\int_{0}^{1} g\left(e^{2 \pi i(\theta+t)}\right) d t=\int_{\theta}^{1+\theta} g\left(e^{2 \pi i t}\right) d t
$$

which is equal to $\int_{0}^{1}$ by periodicity.

Now we are ready to project $C^{*}(E)$ onto the fixed point algebra $C^{*}(E)^{\gamma}$ by averaging over $\gamma$ using [10, Propsition 3.2].

Lemma 3.2.3. Let $\alpha$ be an action of $\mathbb{T}$ on a $C^{*}$-algebra, and define $\Phi: A \rightarrow A$ by

$$
\Phi(a)=\int_{\mathbb{T}} \alpha_{z}(a) d z .
$$

Then $\Phi(a) \in A^{\alpha}$ for every $a \in A$, and $\Phi(a)=$ a for every $a \in A^{\alpha}$. The map $\Phi$ is linear and norm-decreasing, and is faithful in the sense that $\Phi\left(a^{*} a\right)=0$ implies $a=0$.

Proof. Fix $w \in \mathbb{T}$ and $a \in A$, then by parts (iii) and (iv) of Lemma 3.2.2 we can see that $\Phi(a) \in A^{\alpha}$ since

$$
\begin{array}{rlr}
\alpha_{w}(\Phi(a)) & =\alpha_{w} \int_{\mathbb{T}} \alpha_{z}(a) d z & \\
& =\int_{\mathbb{T}} \alpha_{w}\left(\alpha_{z}(a)\right) d z & \\
& =\int_{\mathbb{T}} \alpha_{w z}(a) d z & \\
& =\int_{\mathbb{T}} \alpha_{z}(a) d z & \text { by Lemma 3.2.2.(iii) } \\
& =\Phi(a) . &
\end{array}
$$

To see that $\Phi(a)=a$ for $\forall \alpha \in A^{\alpha}$, fix $a \in A^{\alpha}$ and by $(i)$ of Lemma 3.2.2

$$
\Phi(a)=\int_{\mathbb{T}} \alpha_{z}(a) d z=\int_{\mathbb{T}} a d z=a \int_{\mathbb{T}} 1 d z=a .
$$

The linearity of $\Phi(a)$ can be seen through the application of Equation (3.1). As for the norm-decreasing property of $\Phi(a)$, using part (ii) of Lemma 3.2.2 
and the fact that automorphisms of $C^{*}$-algebras are norm-preserving we can see that

$$
\|\Phi(a)\|=\left\|\int_{\mathbb{T}} \alpha_{z}(a) d z\right\| \leq \int_{\mathbb{T}}\left\|\alpha_{z}(a)\right\| d z=\int_{\mathbb{T}}\|a\| d z=\|a\| .
$$

Lastly, to see that $\Phi$ is faithful, suppose that $\Phi\left(a^{*} a\right)=0$, and let $\pi$ be a faithful representation of $A$. Then for every $h \in \mathcal{H}$ we have

$$
\begin{aligned}
0 & =\left(\pi\left(\Phi\left(a^{*} a\right)\right) h \mid h\right) \\
& =\int_{\mathbb{T}}\left(\pi\left(\alpha_{z}\left(a^{*} a\right)\right) h \mid h\right) d z \\
& =\int_{\mathbb{T}}\left(\pi\left(\alpha_{z}(a)\right)^{*}\left(\pi\left(\alpha_{z}(a)\right) h \mid h\right) d z\right. \\
& =\int_{\mathbb{T}}\left(\pi\left(\alpha_{z}(a)\right) h \mid \pi\left(\alpha_{z}(a)\right) h\right) d z \\
& =\int_{\mathbb{T}}\left\|\pi\left(\alpha_{z}(a)\right) h\right\|^{2} d z .
\end{aligned}
$$

Since $z \mapsto\left\|\pi\left(\alpha_{z}(a)\right) h\right\|^{2}$ is a non-negative continuous function the mapping is zero and $\pi\left(\alpha_{z}(a)\right) h=0$ for all $h \in \mathcal{H}$. Since $\pi$ is faithful, $a=0$.

For the remainder of this thesis $\Phi$ will denote the linear map of $C^{*}(E)$ onto

$$
C^{*}(E)^{\gamma}:=\left\{a \in C^{*}(E): \gamma_{z}(a)=a, \forall z \in \mathbb{T}\right\}
$$

which we call the core of $C^{*}(E)$. We use the core, $C^{*}(E)^{\gamma}$, to create a $\mathbb{Z}$ grading of $C^{*}(E)$. In the following corollary, which follows [10, Corollary 3.3], we show that $C^{*}(E)^{\gamma}=\overline{L_{\mathbb{C}}(E)_{0}}$ before adapting $\Phi$ to project $C^{*}(E)$ onto the subspaces $\overline{L_{\mathbb{C}}(E)_{n}}$.

Corollary 3.2.4. For every finite subset $F$ of $E^{*}$ and every choice of scalars $c_{\mu, \nu}$ we have

$$
\Phi\left(\sum_{\mu, \nu \in F} c_{\mu, \nu} s_{\mu} s_{\nu}^{*}\right)=\sum_{\{\mu, \nu \in F:|\mu|=|\nu|\}} c_{\mu, \nu} s_{\mu} s_{\nu}^{*}
$$

and

$$
C^{*}(E)^{\gamma}=\overline{\operatorname{span}}\left\{s_{\mu} s_{\nu}^{*}:|\mu|-|\nu|=0\right\} .
$$


Proof. Since $\Phi$ is linear we need only consider $\Phi\left(s_{\mu} s_{\nu}^{*}\right)$ for $\mu, \nu \in E^{*}$. If $s(\mu) \neq s(\nu)$ then $\Phi\left(s_{\mu} s_{\nu}^{*}\right)=0$. Else

$$
\begin{aligned}
\Phi\left(s_{\mu} s_{\nu}^{*}\right) & =\int_{\mathbb{T}} \gamma_{z}\left(s_{\mu} s_{\nu}^{*}\right) d z \\
& =\int_{\mathbb{T}} z^{|\mu|-|\nu|} s_{\mu} s_{\nu}^{*} d z \\
& =\left(\int_{\mathbb{T}} z^{|\mu|-|\nu|} d z\right) s_{\mu} s_{\nu}^{*} \\
& = \begin{cases}s_{\mu} s_{\nu}^{*} & \text { if }|\mu|=|\nu| \\
0 & \text { else. }\end{cases}
\end{aligned}
$$

Which proves the first equality.

To show $C^{*}(E)^{\gamma}=\overline{\operatorname{span}}\left\{s_{\mu} s_{\nu}^{*}:|\mu|-|\nu|=0\right\}$, first we check that $\overline{\operatorname{span}}\left\{s_{\mu} s_{\nu}^{*}:|\mu|-|\nu|=0\right\} \subseteq C^{*}(E)^{\gamma}$. Let $a \in \overline{\operatorname{span}}\left\{s_{\mu} s_{\nu}^{*}:|\mu|-|\nu|=0\right\}$, then $a$ is fixed by $\gamma_{z}$ for every $z \in \mathbb{T}$ as $a$ is the limit of sums of elements $s_{\mu} s_{\nu}^{*}$ with $|\mu|=|\nu|$.

We have that $\Phi\left(C^{*}(E)^{\gamma}\right)=C^{*}(E)^{\gamma}$ from Lemma 3.2.3 and by Equation (3.2) $\Phi\left(C^{*}(E)^{\gamma}\right) \subseteq \overline{\operatorname{span}}\left\{s_{\mu} s_{\nu}^{*}:|\mu|-|\nu|=0\right\}=\overline{L_{\mathbb{C}}(E)_{0}}$.

Proposition 3.2.5. Let $E$ be a directed row-finite graph. Then $C^{*}(E)$ is a $\mathbb{Z}-$ graded $C^{*}$-algebra such that

$$
C^{*}(E)=\overline{\bigoplus_{n \in \mathbb{Z}} C^{*}(E)_{n}} .
$$

Proof. This proof follows closely to the proof of [1, Lemma 5.2.11]. For each $n \in \mathbb{Z}$ define

$$
C^{*}(E)_{n}:=\overline{L_{\mathbb{C}}(E)_{n}} .
$$

By Corollary 3.2.4 $\Phi$ maps $C^{*}(E)$ onto $C^{*}(E)^{\gamma}=\overline{L_{\mathbb{C}}(E)_{0}}=C^{*}(E)_{0}$. Define $\Phi_{n}(a):=\int_{\mathbb{T}} z^{-n} \gamma_{z}(a)$. Then

$$
\Phi\left(s_{\mu} s_{\nu}^{*}\right)=\left(\int_{\mathbb{T}}\left(z^{-n}\right)\left(z^{|\mu|-|\nu|} d z\right)\right) s_{\mu} s_{\nu}^{*}= \begin{cases}s_{\mu} s_{\nu}^{*} & \text { if }|\mu|-|\nu|=n \\ 0 & \text { else. }\end{cases}
$$


Which implies

$$
\Phi_{n}\left(\sum_{\mu, \nu \in F} c_{\mu, \nu} s_{\mu} s_{\nu}^{*}\right)=\sum_{\{\mu, \nu \in F:|\mu|-|\nu|=n\}} c_{\mu, \nu} s_{\mu} s_{\nu}^{*},
$$

from which we conclude that $\Phi_{n}$ is a map of $C^{*}(E)$ onto $C^{*}(E)_{n}$.

To see that $C^{*}(E)$ is $\mathbb{Z}$-graded, fix $n, m \in \mathbb{Z}, a_{n} \in C^{*}(E)_{n}$ and $a_{m} \in$ $C^{*}(E)_{m}$. Then

$$
\begin{aligned}
& a_{n} a_{m}=\Phi_{n}\left(a_{n}\right) \Phi\left(a_{m}\right) \\
& =\int_{\mathbb{T}} z^{-n} \gamma_{z}\left(a_{n}\right) d z \cdot \int_{\mathbb{T}} w^{-m} \gamma_{w}\left(a_{m}\right) d w \\
& =\int_{\mathbb{T}} z^{-n} \gamma_{z}\left(a_{n}\right)\left(\int_{\mathbb{T}}\left(z z^{-1} w\right)^{-m} \gamma_{z z^{-1} w}\left(a_{m}\right) d w\right) d z \\
& =\int_{\mathbb{T}} z^{-n} \gamma_{z}\left(a_{n}\right)\left(\int_{\mathbb{T}} z^{-m}\left(z^{-1} w\right)^{-m} \gamma_{z} \circ \gamma_{z^{-1} w}\left(a_{m}\right) d w\right) d z \\
& =\int_{\mathbb{T}} z^{-n} \gamma_{z}\left(a_{n}\right)\left(z^{-m} \gamma_{z}\left(\int_{\mathbb{T}}\left(z^{-1} w\right)^{-m} \gamma_{z^{-1} w}\left(a_{m}\right) d w\right)\right) d z \text { by 3.2.2.(iii) } \\
& =\int_{\mathbb{T}} z^{-n} \gamma_{z}\left(a_{n}\right)\left(z^{-m} \gamma_{z}\left(\int_{\mathbb{T}} w^{-m} \gamma_{w}\left(a_{m}\right) d w\right)\right) d z \quad \text { by 3.2.2.(iv) } \\
& =\int_{\mathbb{T}} z^{-n} \gamma_{z}\left(a_{n}\right) z^{-m} \gamma_{z}\left(a_{m}\right) d z \\
& =\int_{\mathbb{T}} z^{-(n+m)} \gamma_{z}\left(a_{n} a_{m}\right) d z .
\end{aligned}
$$

Since $a_{n}$ and $a_{m}$ were arbitrary, and $\Phi_{m+n}(a) \in C^{*}(E)_{m+n}$ for all $a \in C^{*}(E)$ we have that $C^{*}(E)_{n} C^{*}(E)_{m} \subseteq C^{*}(E)_{n+m}$.

We also have

$$
\begin{aligned}
a_{n}^{*} & =\Phi_{n}\left(a_{n}\right) \\
& =\left(\int_{\mathbb{T}} z^{-n} \gamma_{z}\left(a_{n}\right) d z\right)^{*} \\
& =\int_{\mathbb{T}} z^{n}\left(\gamma_{z}\left(a_{n}\right)\right)^{*} d z \\
& =\int_{\mathbb{T}} z^{n} \gamma_{z}\left(a_{n}^{*}\right) d z
\end{aligned}
$$


for $a_{n} \in C^{*}(E)_{n}$. This implies that $a_{n}^{*} \in C^{*}(E)_{-n}$ and $\left(C^{*}(E)_{n}\right)^{*} \subseteq C^{*}(E)_{-n}$.

Lastly, we need to show that these subspaces are linearly independent. For $a \in C^{*}(E)_{n}$ with $n \neq 0$ we have

$$
\begin{array}{rlr}
\Phi(a) & =\int_{\mathbb{T}} \gamma_{z}(a) d z \\
& =\int_{\mathbb{T}} \gamma_{z}\left(\int_{\mathbb{T}} w^{-n} \gamma_{w}(a) d w\right) d z \\
& =\int_{\mathbb{T}} \gamma_{z}\left(\int_{\mathbb{T}}\left(z^{-1} z w\right)^{-n} \gamma_{z^{-1} z w}(a) d w\right) d z & \\
& =\int_{\mathbb{T}} \gamma_{z}\left(z^{n} \gamma_{z^{-1}} \int_{\mathbb{T}}(z w)^{-n} \gamma_{z w}(a) d w\right) d z \quad \text { by 3.2.2.(iii) } \\
& =\int_{\mathbb{T}} z^{-n} \gamma_{z z^{-1}}\left(\int_{\mathbb{T}}(w)^{-n} \gamma_{w}(a) d w\right) d z \quad \text { by 3.2.2.(iv) } \\
& =\int_{\mathbb{T}} z^{-n} a d z \\
& =0 .
\end{array}
$$

Suppose $\sum_{i=-N}^{N} a_{i}=0$ for some $N \in \mathbb{Z}$ with $a_{i} \in C^{*}(E)_{i}$ for each $i$. Then

$$
\left(\sum_{i=-N}^{N} a_{i}\right)^{*}=\sum_{i=-N}^{N} a_{i}^{*}
$$

Fix $-N<j<N$. Then $a_{j}^{*} \in C^{*}(E)_{-j}$ since $\left(C^{*}(E)_{j}\right)^{*} \subseteq C^{*}(E)_{-j}$ and $a_{j}^{*} a_{i} \in$ $C^{*}(E)_{-j+i}$ for each $-N \leq i \leq N$ because $C^{*}(E)_{-j} C^{*}(E)_{i} \subseteq C^{*}(E)_{-j+i}$. Therefore

$$
0=\Phi\left(\sum_{i=-N}^{N} a_{j}^{*} a_{i}\right)=\sum_{i=-N}^{N} \Phi\left(a_{j}^{*} a_{i}\right)=\Phi\left(a_{j}^{*} a_{j}\right) .
$$

Since $a_{j}^{*} a_{i} \notin C^{*}(E)^{\gamma}, a_{j}^{*} a_{i}=0$ for $i \neq j$. Thus, $a_{j}=0$ because $\Phi$ is faithful. Therefore the subspaces $C^{*}(E)_{n}$ are linearly independent and $C^{*}(E)$ is $\mathbb{Z}$ graded. 


\subsection{The Core}

In Section 3.2 we saw that the gauge action maps $C^{*}(E)$ onto the fixed point algebra $C^{*}(E)^{\gamma}=\overline{\operatorname{span}}\left\{s_{\mu} s_{\nu}^{*}:|\mu|-|\nu|=0\right\}$, which we called the core of $C^{*}(E)$. In the context of grading, $C^{*}(E)^{\gamma}$ is $C^{*}(E)_{0}$. In fact, the subspace $A_{e}$ of any $G$-graded algebra $A$, where $e$ is the identity of $G$, forms a subalgebra of $A$; since it is closed under addition by liberty of its span and according to the definition of grading we have that $A_{e} \cdot A_{e} \subseteq A_{e}$ so it is closed under multiplication.

We have seen that $L_{\mathbb{C}}(E)$ is dense in $C^{*}(E)$ and that $L_{\mathbb{C}}(E)_{0}$ sits densely inside $C^{*}(E)^{\gamma}$. Since $\Phi\left(L_{\mathbb{C}}(E)\right)=L_{\mathbb{C}}(E)_{0}$ we will refer to $L_{\mathbb{C}}(E)_{0}$ as the core of $L_{\mathbb{C}}(E)$. Both $L_{\mathbb{C}}(E)_{0}$ and $C^{*}(E)^{\gamma}$ are isomorphic to the direct limit of matricial algebras, a fact we rely heavily upon in the proof of our main result.

We begin with a short summary of section A.2. of [10] which discusses matrix algebras; then we apply these results to the core of both the Leavitt path algebra and graph $C^{*}$-algebra of a row-finite graph $E$ taking heavy direction from the discussion of the structure of $C^{*}(E)^{\gamma}$ in chapter 3 of [10].

A matrix unit is a matrix $E_{i j} \in M_{n}(\mathbb{C})$ whereby $1 \leq i, j \leq n$ and

$$
\left(E_{i j}\right)_{k l}= \begin{cases}1 & \text { if } i=k, j=l \\ 0 & \text { else }\end{cases}
$$

Every $a \in M_{n}(\mathbb{C})$ can be written as $\sum_{i, j} a_{i j} E_{i j}$ and hence the set $\left\{E_{i j}\right\}$ forms a basis for $M_{n}(\mathbb{C})$.

We can also see, through simple calculations, that the matrix units $E_{i j}$ are such that

$$
E_{i j}^{*}=E_{j i} \quad \text { and } \quad E_{i j} E_{k l}= \begin{cases}E_{i l} & \text { if } j=k \\ 0 & \text { else }\end{cases}
$$


We use these conditions to find copies of $M_{n}(\mathbb{C})$ in our algebras by finding families satisfying the necessary relations. In [10, Proposition A.5] Raeburn shows that for a $C^{*}$-algebra $B$ with a non-zero family $\left\{e_{i j}: 1 \leq i, j \leq\right.$ $n\}$ satisfying the above relations there is an injective homomorphism $\phi$ from $M_{n}(\mathbb{C})$ in to $B$ such that $\phi\left(E_{i j}\right)=e_{i j}$.

Suppose $E$ is a row-finite directed graph. We use the discussion above to analyse the structure of the core of both $L_{\mathbb{C}}(E)$ and $C^{*}(E)$. For a given $k \in \mathbb{N}$, define

$$
\mathcal{F}_{k}:=\operatorname{span}\left\{s_{\mu} s_{\nu}^{*}: \mu, \nu \in E^{k}\right\}
$$

For any paths $\mu, \nu, \alpha, \beta \in E^{k}$, we have

$$
\left(s_{\mu} s_{\nu}^{*}\right)\left(s_{\alpha} s_{\beta}^{*}\right)= \begin{cases}s_{\mu} s_{\beta}^{*} & \text { if } \nu=\alpha \\ 0 & \text { else }\end{cases}
$$

by Lemma 2.2.4 and the fact that $|\nu|=|\alpha|=k$. Moreover, we have that $\left(s_{\mu} s_{\nu}^{*}\right)^{*}=s_{\nu} s_{\mu}^{*}$; hence $\mathcal{F}_{k}$ is a family of matrix units.

For every $v \in E^{0}$,

$$
\mathcal{F}_{k}(v):=\operatorname{span}\left\{s_{\mu} s_{\nu}^{*}: \mu, \nu \in E^{k}, s(\mu)=s(\nu)=v\right\}
$$

is a family of matrix units we can see this using Equation (3.3) in conjunction with [10, Proposition A.5]. For a row-finite graph there are a finite number of paths $\mu \in E^{k} \cap s^{-1}(v)$ so $\mathcal{F}_{k}(v) \cong M_{\left|E^{k} \cap s^{-1}(v)\right|}(\mathbb{C})$.

Since no path of $\mathcal{F}_{k}(v)$ is in $\mathcal{F}_{k}(w)$ for all $v, w \in E^{0}$ such that $v \neq w$ we have $\mathcal{F}_{k}(v) \mathcal{F}_{k}(w)=0$. Hence

$$
\mathcal{F}_{k}=\bigoplus_{v \in E^{0}} \mathcal{F}_{k}(v)
$$

When the graph $E$ has no sources, the Cuntz-Krieger relation at $v$ gives

$$
s_{\mu} s_{\nu}^{*}=s_{\mu} p_{v} s_{\nu}^{*}=\sum_{r(e)=v} s_{\mu} s_{e} s_{e}^{*} s_{\nu}^{*}=\sum_{r(e)=v} s_{\mu e} s_{\nu e}^{*}
$$


which implies that $\mathcal{F}_{k} \subset \mathcal{F}_{k+1}$. So

$$
\cup_{k}\left(\oplus_{v \in E^{0}} \mathcal{F}_{k}(v)\right)=\cup_{k} \mathcal{F}_{k}=\operatorname{span}\left\{s_{\mu} s_{\nu}^{*}: \mu, \nu \in E^{*},|\mu|=|\nu|\right\}=L_{\mathbb{C}}(E)_{0}
$$

and

$$
\overline{\cup_{k}\left(\oplus_{v \in E^{0}} \mathcal{F}_{k}(v)\right)}=\overline{\cup_{k} \mathcal{F}_{k}}=\overline{\operatorname{span}}\left\{s_{\mu} s_{\nu}^{*}: \mu, \nu \in E^{*},|\mu|=|\nu|\right\}=C^{*}(E)^{\gamma} .
$$

Thus, the core of both $L_{\mathbb{C}}(E)$ and $C^{*}(E)$ are isomorphic to the direct limit of matrix algebras.

For general row-finite graphs Raeburn uses a technique introduced by Yeend (2003) to analyse the core of $C^{*}(E)$ which we can also use for the core of $L_{\mathbb{C}}(E)$. Take $k \in \mathbb{N}$, we define

$$
E^{\leq k}:=\left\{\mu \in E^{*}:|\mu|=k \text {, or }|\mu|<k \text { and } s(\mu) \text { is a source }\right\} .
$$

If $\nu, \alpha \in E^{\leq k}$ and $|\nu|<|\alpha|$, then $\nu \neq \alpha \nu^{\prime}$. Additionally $|\nu|<k$ and $s(\nu)$ is a source of $E$ meaning that $\alpha$ cannot extend $\nu$. Thus by Lemma 2.2.4 $\left(s_{\mu} s_{\nu}^{*}\right)\left(s_{\alpha} s_{\beta}^{*}\right)=0$. By the same reasoning, if $|\alpha|<|\nu|$ then $\alpha \neq \nu \alpha^{\prime}$ and $\nu \neq \alpha \nu^{\prime}$. Hence Equation (3.3) holds for all $\mu, \nu, \alpha, \beta \in E^{\leq k}$. Thus

$$
\mathcal{F}_{\leq k}(v):=\operatorname{span}\left\{s_{\mu} s_{\nu}^{*}: \mu, \nu \in E^{\leq k} \text { and } s(\mu)=s(\nu)=v\right\}
$$

is a family of matrix units and

$$
\mathcal{F}_{\leq k}:=\operatorname{span}\left\{s_{\mu} s_{\nu}^{*}: \mu, \nu \in E^{\leq k}\right\}
$$

is the direct $\bigoplus_{v \in E^{0}} \mathcal{F}_{\leq k}(v)$. If $v$ is not a source then all paths $\mu, \nu \in E^{\leq k}$ must have length $k$, and we know that $\mathcal{F}_{k} \subset \mathcal{F}_{k+1}$ from above; thus

$$
\mathcal{F}_{\leq k}(v)=\mathcal{F}_{k}(v) \subset \mathcal{F}_{k} \subset \mathcal{F}_{k+1} \subset \mathcal{F}_{\leq k+1}
$$

If $v$ is a source then from the definition of $E^{\leq k}$ we can see that

$$
\left(E^{\leq k} \cap s^{-1}(v)\right) \subset\left(E^{\leq k+1} \cap s^{-1}(v)\right)
$$


and thus $\mathcal{F}_{\leq k}(v) \subset \mathcal{F}_{\leq k+1}(v)$. As such $\mathcal{F}_{\leq k} \subset \mathcal{F}_{\leq k+1}$, giving us that

$$
L_{\mathbb{C}}(E)_{0} \subset \cup_{k} \mathcal{F}_{\leq k}=\cup_{k}\left(\oplus_{v \in E^{0}} \mathcal{F}_{\leq k}\right)
$$

and

$$
C^{*}(E)^{\gamma} \subset \overline{\cup_{k} \mathcal{F}_{\leq k}}=\overline{\cup_{k}\left(\oplus_{v \in E^{0}} \mathcal{F}_{\leq k}\right)}
$$

If $E$ has sources the inclusion will be proper, because $\mathcal{F}_{\leq k}(v)$ will include elements $s_{\mu} s_{\nu}^{*}$ such that $|\mu| \neq|\nu|$. If we want to describe the core of $L_{\mathbb{C}}(E)$ and $C^{*}(E)$ we will need to introduce the sub-algebras

$$
\mathcal{F}_{k, l}(v):=\operatorname{span}\left\{s_{\mu} s_{\nu}^{*}: \mu, \nu \in E^{\leq k},|\mu|=|\nu|=l, s(\mu)=s(\nu)=v\right\} .
$$

Again we can see that $\mathcal{F}_{k, l}(v)$ contains a family of matrix units. Then

$$
\mathcal{G}_{k}(v):=\mathcal{F}_{\leq k}(v) \cap L_{\mathbb{C}}(E)_{0}=\bigoplus_{l=1}^{k} \mathcal{F}_{k, l}(v),
$$

and

$$
\mathcal{G}_{k}:=\mathcal{F}_{\leq k} \cap L_{\mathbb{C}}(E)_{0}=\bigoplus_{v \in E^{0}} \mathcal{G}_{k}(v) .
$$

Since $\mathcal{F}_{\leq k} \subset \mathcal{F}_{\leq k+1}$ and $L_{\mathbb{C}}(E)_{0}$ is closed under multiplication we have that $\mathcal{G}_{k} \subset \mathcal{G}_{k+1}$. From this we can see that

$$
L_{\mathbb{C}}(E)_{0}=\bigcup_{k}\left(\bigoplus_{v \in E^{0}} \mathcal{G}_{k}(v)\right)
$$

and

$$
C^{*}(E)^{\gamma}=\overline{\bigcup_{k}\left(\bigoplus_{v \in E^{0}} \mathcal{G}_{k}(v)\right)}
$$

\subsection{Fell Bundles}

In this section we discuss the structure of Fell Bundles that can be found within a $C^{*}$ algebra and their relation to gradings. The definitions and results of this section can be found in Chapter 16 of [5]. 
A Fell bundle over a group $G$ is a collection of Banach spaces

$$
\mathscr{B}=\left\{B_{g}\right\}_{g \in G},
$$

wherein each $B_{g}$ is known as a fibre. In addition, the disjoint union of these fibres, known as the total space of $\mathscr{B}$, is equipped with a multiplication operation and an involution satisfying the following properties for all $g, h \in G$ and $b, c \in \mathscr{B}$ :

(i) $B_{g} B_{h} \subseteq B_{g h}$;

(ii) multiplication is bilinear from $B_{g} \times B_{h}$ to $B_{g h}$;

(iii) multiplication on $\mathscr{B}$ is associative;

(iv) $\|b c\| \leq\|b\|\|c\|$;

(v) $\left(B_{g}\right)^{*} \subseteq B_{g^{-1}}$;

(vi) involution is conjugate linear from $B_{g}$ to $B_{g^{-1}}$;

(vii) $(b c)^{*}=c^{*} b^{*}$;

(viii) $b^{* *}=b$;

(ix) $\left\|b^{*}\right\|=\|b\|$;

(x) $\left\|b^{*} b\right\|=\|b\|^{2}$;

(xi) $b^{*} b \geq$ in $B_{e}$.

According to [5], the properties (i) to (iv) give rise to what is known as a Banach Algebraic Bundle, and with the addition of (v) to (x) describe a Banach *algebraic Bundle.

Given that $B_{e} \times B_{e}$ maps to $B_{e}$ and $\left(B_{e}\right)^{*} \subseteq B_{e}$, we can observe that axioms (i) through (x) imply that $B_{e}$ is a $C^{*}$-algebra using given operations. We often call $B_{e}$ the unit fibre algebra. 
Remark 3.4.1. Clearly, if given a $G$-graded $C^{*}$-algebra $B$ the grading subspaces $B_{g}$ form a Fell bundle with respect to the norm, multiplication and adjoint of $B$. However, $B$ cannot necessarily be uniquely determined from $\mathscr{B}$, because there can be multiple ways to complete $\mathscr{B}$.

Before we move on we will visit two examples which illustrate this point, and introduce a notion of grading for $C^{*}$-algebras with stronger conditions.

Example 3.4.2. This first example is discussed in [12, Example 1]. Consider the graph $E$ consisting of a single vertex $v$ and a single loop $e$. The CuntzKrieger E-family $\{S, P\}$ of $\mathrm{E}$ is generated by the single unitary operator $S_{e}$, and $P_{v}$ is an identity for $C^{*}(E)$. Since graph algebras are universal for Cuntz-Krieger E-families, this graph is universal for all $C^{*}$-algebras generated by a single unitary element, and since for any $\mathrm{C}^{*}$-algebra $B$ generated by a unitary element $u$ we can construct the homomorphism $\pi_{u}: C^{*}(E) \rightarrow B$ so that $\pi_{u}\left(S_{e}\right)=u$.

Consider $\mathbb{T}$ and the identity function $\iota: z \mapsto z$ from $\mathbb{T}$ to $\mathbb{C}$. Now $\iota$ is unitary, and the $C^{*}$-algebra generated by $\iota$ is the closed span of the functions $\iota^{m}\left(\iota^{*}\right)^{n}: z \mapsto z^{m} \bar{z}^{n}$, which for $\mathbb{T}$ is the polynomials $z^{k}$. Moreover, the Stone-Weierstrass Theorem tells us that $C^{*}(\iota)=C(\mathbb{T})$. Hence $\left(C^{*}(E), S_{e}\right)$ is $(C(\mathbb{T}), z)$.

We know from Proposition 3.2.5 that $C^{*}(E)$ is a $\mathbb{Z}$-graded algebra, and we can observe the grading $C(\mathbb{T})_{n}$ by considering the span of polynomials $z^{n}$. As discussed above, the subspaces form a Fell bundle $\mathscr{B}$, with fibres $B_{n}$ consisting of the polynomials $z^{n}$. We can see that the polynomials $z^{n}$ form an orthonormal basis for $L^{2}(\mathbb{T})$ and $C(\mathbb{T}) \subset L^{2}(\mathbb{T})$, so the Fourier coefficients

$$
\hat{f}(n)=\int_{\mathbb{T}} f(z) z^{-n} d z:=\int_{0}^{1} f\left(e^{2 \pi i t}\right) e^{-2 \pi i t} d t
$$

of $f \in C(\mathbb{T})$ determine $f$ uniquely and $\hat{f}(n)=\hat{g}(n)$ for all $n$ implies $f=g$ in $C(\mathbb{T})$. A classical result of Fejér (1900) tells us that the Cesàro means (we 
say that a sequence $\left\{a_{n}\right\}$ is Cesàro summable, with Cesàro sum $A \in \mathbb{R}$, if as $n$ tends to infinity the arithmetic means of the partial sums $s_{k}:=$ $a_{1}+a_{2}+\ldots+a_{k}=\sum_{n=1}^{k} a_{n}$ converge to $A$, ie, $\lim _{n \rightarrow \infty} \frac{1}{n} \sum_{k=1}^{n} s_{k}=A$ ) of the partial sums of the Fourier series converge uniformly to $f$ on $\mathbb{T}$. As such we can recover $f$ from its Fourier coefficients which allows us to view $C(\mathbb{T})$ as a $\mathbb{Z}$-graded algebra.

Example 3.4.3. This second example is discussed in both [5, p. 156], and [12, Example 2]. In this example we take a closed proper subset $X$ of $\mathbb{T}$ which is infinite, and consider $C(X)$ generated by the inclusion map $\iota: X \rightarrow \mathbb{C}$ whereby $z \mapsto z$, we say that $C(X)$ is generated by $z$. Now we can consider whether $B_{n}=\mathbb{C} z^{m}, \forall n \in \mathbb{Z}$ can constitute a $\mathbb{Z}$-grading for $C(X)$.

Clearly, we can see that $B_{n} B_{m} \subseteq B_{n+m} \forall m, n \in \mathbb{Z}$, and $B_{n}^{*}=B_{-n}$ for every $n \in \mathbb{Z}$. Now we must check that the $B_{n}$ 's are linearly independent subspaces of $C(X)$. To see this suppose that $\sum_{n \leq N} c_{n} z^{n}=0$ on $X$. Then

$$
\iota(z)=\sum_{n \leq N} c_{n} z^{n}=0, \quad \forall z \in X
$$

Then $z^{-n} \iota(z)=z^{-N} p(z)$, for some polynomial $p$ of degree $2 N$. Now, $\left|z^{-1}\right| \neq 0$, so $p(z)=0$ for all $z$ in $X$, which, by the fundamental theorem of algebra, implies that $X$ is contained in the roots of $p$, and $|X| \leq 2 N$. However, $X$ was chosen as an infinite set, therefore $c_{n}=0$ for each $n$ and the subspaces $C(X)_{n}$ are linearly independent.

Now, $X$ is a proper closed subset of $\mathbb{T}$, so $X$ is compact; meaning each continuous function $f \in C(X)$ is bounded. We can apply Tietze's Extension Theorem, as described in [2, Theorem 3.2.13], to the real and imaginary parts of $f$ to see that there exists a continuous function $g: \mathbb{T} \rightarrow \mathbb{C}$ such that $g_{\left.\right|_{X}}=f$, for all $x \in X$. So the map $g \mapsto g_{\left.\right|_{X}}$ is onto.

Each $f \in C(X)$ has many extensions $g \in C(\mathbb{T})$. As discussed in the previous example each such extension $g$ has a canonical sequence of homogeneous components $\hat{g}(n)$, over which the Cesàro means of the partial sums 
converge uniformly to $g$ in $C(\mathbb{T})$. The restrictions of the Cesàro means to $X$ converge uniformly in $C(X)$ to $g_{\left.\right|_{X}}=f$. However, each extension of $f$ will have different Fourier coefficients, and there is no canonical way to choose homogeneous components for $f$ in $C(X)$.

These examples demonstrate the strong connection between graded $C^{*}$-algebras and Fell Bundles. In Example 3.4.2 we see that we can derive $C(\mathbb{T})$ from the Fell bundle $\mathscr{B}$, but we are unable to obtain $C(X)$ from the Fell bundle made up of the spaces $C(X)_{n}$ as described in Example 3.4.3. When we can reassemble a graded $C^{*}$-algebra from its parts we say that it is topologically graded. More formally we say a grading on a $C^{*}$-algebra $B$ is said to be a topological grading if there exists a (necessarily unique) conditional expectation of $B$ onto $B_{e}$, vanishing on all $B_{g}$ for $g \neq e$.

Now we move on to discuss some properties of Fell bundles and a class of Fell bundles known as saturated Fell bundles as they hold particular significance to our result.

Lemma 3.4.4. For any Fell Bundle $\mathscr{B}=\left\{B_{g}\right\}_{g \in G}$ and $g \in G, \overline{B_{g} B_{g^{-1}}}$ is a closed ideal of $B_{e}$ (where $\overline{B_{g} B_{h}}$ is the closed span of $B_{g} B_{h}$ ).

Proof. Fix $g \in G$, then by continuity, we have

$$
\overline{B_{g} B_{g^{-1}}} B_{e} \subseteq \overline{B_{g} B_{g^{-1}} B_{e}} \subseteq \overline{B_{g} B_{g^{-1}}}
$$

and

$$
B_{e} \overline{B_{g} B_{g^{-1}}} \subseteq \overline{B_{e} B_{g} B_{g^{-1}}} \subseteq \overline{B_{g} B_{g^{-1}}}
$$

If $\mathscr{B}=\left\{B_{g}\right\}_{g \in G}$ is a Fell Bundle with $\overline{B_{g} B_{h}}=B_{g h}$ for all $g, h \in G$, we say that $\mathscr{B}$ is saturated as per the definition given in [5, Definition 16.10]. This definition seems to hold certain parallels with that of strong grading, a connection which we intend to demonstrate in our main result. But first, we need to better understand the structure of a saturated Fell Bundle. 
Proposition 3.4.5. A Fell bundle $\mathscr{B}=\left\{B_{g}\right\}_{g \in G}$ is saturated if and only if $\overline{B_{g} B_{g^{-1}}}=B_{e} \forall g \in G$.

We present the proof of Proposition 3.4.5 after the following Lemmas. We have $\overline{B_{g} B_{g^{-1}}} \subseteq B_{e}$ for any $g \in G$ by definition; however, we need to do more work to show that $B_{e} \subseteq B_{g} B_{g^{-1}}$ using Bi-modules which we describe below using [5, Definition 15.1].

Given a $C^{*}$-algebra $A$, we say that an right pre-Hilbert $A$-module is a complex linear space $M$ equipped with a right $A$-module structure with a $\operatorname{map}\langle\cdot, \cdot\rangle: M \times M \rightarrow A$ satisfying the following properties for all $x, y, z \in$ $M, \alpha, \beta \in \mathbb{C}$, and $a \in A$ :

(i) $\langle x, \alpha y+\beta z\rangle=\alpha\langle x, y\rangle+\beta\langle x, z\rangle$;

(ii) $\langle x, y a\rangle=\langle x, y\rangle$;

(iii) $\langle x, y\rangle^{*}=\langle y, x\rangle$;

(iv) $\langle x, x\rangle \geq 0$ and $\langle x, x\rangle=0 \Longleftrightarrow x=0$.

We define a left pre-Hilbert A-module similarly, where we assume $M$ to be have a left $A$-module structure and we alter the first two axioms to get:

$\left(\mathrm{i}^{\prime}\right)\langle\alpha x+\beta y, z\rangle=\alpha\langle x, z\rangle+\beta\langle y, z\rangle ;$

(ii') $\langle a x, y\rangle=a\langle x, y\rangle$.

We can define a norm over the pre-Hilbert $A$-module $M$ whereby $\|m\|=$ $\|\langle m, m\rangle\|^{1 / 2}$. If $M$ is complete with respect to this norm we say that $M$ is a Hilbert A-module.

Lemma 3.4.6. If $\left\{v_{i}\right\}$ is an approximate identity for $B_{e}$, then for any $g \in G$ and $b \in B_{g}$,

$$
b=\lim _{i} b v_{i}=\lim _{i} v_{i} b
$$


Proof. Fix $g \in G$, we claim that $B_{g}$ is a Hilbert $B_{e}-B_{e}$-bimodule. Clearly $B_{g}$ forms a $B_{e}$-bimodule and through the associativity of multiplication over $\mathscr{B}$ together with the sesquilinear maps giving $\langle x, y\rangle_{R}:=x y^{*}$ from the right and $\langle x, y\rangle_{L}:=x^{*} y$ from the left which map from $B_{g} \times B_{g}$ to $B_{e}$ since $B_{g}^{*} B_{g} \subseteq B_{e}$ and $B_{g} B_{g}^{*} \subseteq B_{e}$. These maps give us that

$$
\langle x, y\rangle_{R} z=\left(x y^{*}\right) z=x\left(y^{*} z\right)=x\langle y, z\rangle_{L} .
$$

The fibre $B_{g}$ will be complete with respect to the norm $\|\cdot\|_{\langle i,\rangle}$ defined over the Hilbert bimodule given that $B_{g}$ is a Banach space and $\|b\|_{\langle\cdot,\rangle}^{2}=$ $\|\langle b, b\rangle\|=\left\|b^{*} b\right\|=\|b\|^{2}$.

Since $B_{g}$ is a Hilbert $B_{e}-B_{e}$-bimodule we can see from the result of [5, Lemma 15.2] that

$$
b=\lim _{i} b v_{i}=\lim _{i} v_{i} b \quad \forall b \in B_{g} .
$$

Lemma 3.4.7. For a Fell bundle $\mathscr{B}=\left\{B_{g}\right\}_{g \in G}$ and any $g \in G, B_{g}=\overline{B_{g} B_{g^{-1}} B_{g}}$.

Proof. Firstly since $B_{g}$ and $B_{g^{-1}}$ are fibres of $\mathscr{B}, \overline{B_{g} B_{g^{-1}} B_{g}} \subseteq \overline{B_{e} B_{g}} \subseteq B_{g}$.

To show the other containment let $\left\{v_{i}\right\}$ be an approximate identity for $\overline{B_{g} B_{g^{-1}}}$ and $\left\{u_{i}\right\}$ be an approximate identity for $\overline{B_{g^{-1}} B_{g}}$. Then for any $b \in$ $B_{g}$

$$
b=\lim _{i} v_{i} b=\lim _{i} b u_{i}
$$

since $B_{g}$ will be a $B_{g} B_{g^{-1}}-B_{g^{-1}} B_{g}$-bimodule using the same sesquilinear forms discussed in Lemma 3.4.6. This implies that $B_{g}$ is a right Hilbert $\overline{B_{g^{-1}} B_{g}}$-module and a left Hilbert $\overline{B_{g} B_{g^{-1}}}$-module. According to [5, Lemma 15.2] this means that $b=\lim _{i} v_{i} b=\lim _{i} b u_{i}$. So $B_{g} \subseteq \overline{B_{g} B_{g^{-1}} B_{g}}$.

The proof of Proposition 3.4.5. As stated earlier if $\overline{B_{g} B_{h}}=B_{g h} \forall g, h \in G$ then $\overline{B_{g} B_{g^{-1}}}=B_{e}$. Suppose that $\overline{B_{g} B_{g^{-1}}}=B_{e}$ for every $g \in G$. We fix $g, h \in G$, 
and compute

$$
\begin{array}{rlr}
B_{g h} & =\overline{B_{g h} B_{(g h)^{-1} B_{g h}}} & (\text { by Lemma 3.4.7) } \\
& \subseteq \overline{B_{e} B_{g h}} & \left(\overline{B_{g} B_{g^{-1}}}=B_{e} \forall g \in G\right) \\
& =\overline{B_{g} B_{g^{-1}} B_{g h}} & \\
& \subseteq \overline{B_{g} B_{h} .}
\end{array}
$$




\section{Chapter 4}

\section{Strongly Graded Graph $\mathrm{C}^{*}$-algebras}

Suppose that a ring $A$ is graded by a group $G$, such that $A=\oplus_{g \in G} A_{g}$. We say that $A$ is strongly graded if $A_{g} \cdot A_{h}=A_{g h}$ for every $g, h \in G$.

Motivated by this definition and the definition of saturated Fell bundles we define strongly graded $\mathrm{C}^{*}$-algebras as follows:

Definition 4.0.1. Let $A$ be a $G$-graded $C^{*}$-algebra with the $C^{*}$-grading $\left\{A_{g}\right\}_{g \in G}$. Then $A$ is strongly G-graded if

$$
\overline{A_{g} \cdot A_{h}}=A_{g h}
$$

for every $g, h \in G$.

If the group $G$ is clear, we say that $A$ is strongly graded.

In Remark 3.4.1 we discussed how the grading of a $C^{*}$-algebra forms a Fell bundle. Any grading of a $\mathrm{C}^{*}$-algebra satisfying the conditions for a strongly graded $C^{*}$-algebra would indeed form a saturated Fell bundle; and if a Fell bundle generated by the grading of a $G$-graded $C^{*}$-algebra $A$ were saturated then $A$ would be strongly graded.

A graph $E$ satisfies Condition $(Y)$ if for every $k \in \mathbb{N}$ and every infinite path $p$, there exists a final subpath $\alpha \in E^{*}$ and $\beta \in E^{*}$ such that $s(\beta)=s(\alpha)$ 
and $|\beta|-|\alpha|=k$ [3, Definition 4.1]. In [3, Theorem 4.2] Clark, Hazrat and Rigby prove that $L_{\mathbb{C}}(E)_{K}(E)$ is strongly graded if and only if $E$ has no sources and satisfies Condition $(\mathrm{Y})$.

The main new result of this thesis is as follows:

Theorem 4.0.2. Suppose $E$ is a row-finite graph. Then $C^{*}(E)$ is a strongly graded $C^{*}$-algebra if and only if E has no sources and satisfies Condition (Y).

We tried to prove this directly by modifying the argument of [3, Theorem 4.2] but found the "unusual thing" of deducing the $C^{*}$-algebra result from the Leavitt path algebra result worked better and was cleaner.

Theorem 4.0.3. Let $E$ be a row-finite graph. Then $C^{*}(E)$ is a strongly graded $C^{*}$-algebra if and only if $L_{\mathbb{C}}(E)$ is a strongly graded ring.

The easier argument is the "if" direction.

Proposition 4.0.4. Suppose $E$ is a row-finite graph. If $L_{\mathbb{C}}(E)$ is a strongly graded ring then $C^{*}(E)$ is a strongly graded $C^{*}$-algebra.

Proof. If $L_{\mathbb{C}}(E)$ is strongly graded then

$$
L_{\mathbb{C}}(E)_{n} \cdot L_{\mathbb{C}}(E)_{m}=L_{\mathbb{C}}(E)_{n+m} \quad \forall n, m \in \mathbb{Z} .
$$

Since $C^{*}(E)$ is graded we have that $C^{*}(E)_{n} \cdot C^{*}(E)_{m} \subseteq C^{*}(E)_{n+m}$ and since $C^{*}(E)_{n+m}$ is a Banach space it is closed; giving us $\overline{C^{*}(E)_{n} \cdot C^{*}(E)_{m}} \subseteq$ $C^{*}(E)_{n+m}$. It remains to show is that $C^{*}(E)_{n+m} \subseteq \overline{C^{*}(E)_{n} \cdot C^{*}(E)_{m}}$. To see this, fix $a \in C^{*}(E)_{n+m}$. Since $C^{*}(E)_{n+m}=\overline{L_{\mathbb{C}}(E)_{n+m}}$ by Proposition 3.2.5, there exists a sequence

$$
\left\{a_{i}\right\} \subseteq L_{\mathbb{C}}(E)_{n+m} \text { such that } a_{i} \rightarrow a \text { as } i \rightarrow \infty \text {. }
$$

Then $\left\{a_{i}\right\} \subseteq L_{\mathbb{C}}(E)_{n} \cdot L_{\mathbb{C}}(E)_{m} \subseteq C^{*}(E)_{n} \cdot C^{*}(E)_{m}$ and hence $a \in \overline{C^{*}(E)_{n} \cdot C^{*}(E)_{m}}$.

For the other direction we prove the contrapositive. 
Proposition 4.0.5. Suppose $E$ is a row-finite graph. If $L_{\mathbb{C}}(E)$ is not a strongly graded ring then $C^{*}(E)$ is not a strongly graded $C^{*}$-algebra.

To prove this we need the following Lemmas:

Lemma 4.0.6. Let $E$ be a graph. Then $L_{\mathbb{C}}(E)_{n} \cdot L_{\mathbb{C}}(E)_{-n}$ is a ${ }^{*}$-subalgebra of $L_{\mathbb{C}}(E)$.

Proof. By definition $L_{\mathbb{C}}(E)_{n} \cdot L_{\mathbb{C}}(E)_{-n}=\operatorname{span}\left\{a b: a \in L_{\mathbb{C}}(E)_{n}, b \in L_{\mathbb{C}}(E)_{-n}\right\}$ so it is closed under addition and scalar multiplication. To see that it is closed under multiplication it suffices to show that two generators of $L_{\mathbb{C}}(E)_{n} \cdot L_{\mathbb{C}}(E)_{-n}$ multiplied remain in $L_{\mathbb{C}}(E)_{n} \cdot L_{\mathbb{C}}(E)_{-n}$ thanks to the distributive property of multiplication. Suppose $a$ and $b$ are two generators of $L_{\mathbb{C}}(E)_{n} \cdot L_{\mathbb{C}}(E)_{-n}$ with $a=a_{n} a_{-n}$ and $b=b_{n} b_{-n}$ such that $a_{n}, b_{n} \in L_{\mathbb{C}}(E)_{n}$ and $a_{-n}, b_{-n} \in L_{\mathbb{C}}(E)_{-n}$. Then

$$
a b=\left(a_{n} a_{-n}\right)\left(b_{n} b_{-n}\right)=a_{n}\left(a_{-n}\left(b_{n} b_{-n}\right)\right) .
$$

Since $L_{\mathbb{C}}(E)$ is $\mathbb{Z}$-graded we have that

$$
a_{-n}\left(b_{n} b_{-n}\right) \in L_{\mathbb{C}}(E)_{-n} \cdot L_{\mathbb{C}}(E)_{n} \cdot L_{\mathbb{C}}(E)_{-n} \subseteq L_{\mathbb{C}}(E)_{-n} \cdot L_{\mathbb{C}}(E)_{0} \subseteq L_{\mathbb{C}}(E)_{-n}
$$

So $a b=a_{n}\left(a_{-n}\left(b_{n} b_{-n}\right)\right) \in L_{\mathbb{C}}(E)_{n} \cdot L_{\mathbb{C}}(E)_{-n}$.

Lastly for *-closure, fix a generator $a=a_{n} a_{-n} \in L_{\mathbb{C}}(E)_{n} \cdot L_{\mathbb{C}}(E)_{-n}$ with $a_{n} \in L_{\mathbb{C}}(E)_{n}$ and $a_{-n} \in L_{\mathbb{C}}(E)_{-n}$ then

$$
a^{*}=\left(a_{n} a_{-n}\right)^{*}=a_{-n}^{*} a_{n}^{*}
$$

and since $L_{\mathbb{C}}(E)_{m}^{*} \subseteq L_{\mathbb{C}}(E)_{-m}$ for all $m \in \mathbb{Z}$, we have

$$
a_{-n}^{*} a_{n}^{*} \in L_{\mathbb{C}}(E)_{n} \cdot L_{\mathbb{C}}(E)_{-n} .
$$

Lemma 4.0.7. Let $E$ be a row-finite graph, then

$$
\overline{L_{\mathbb{C}}(E)_{n} \cdot L_{\mathbb{C}}(E)_{-n}}=\overline{C^{*}(E)_{n} \cdot C^{*}(E)_{-n}} .
$$


Proof. We have $L_{\mathbb{C}}(E)_{n} \cdot L_{\mathbb{C}}(E)_{-n} \subseteq C^{*}(E)_{n} \cdot C^{*}(E)_{-n}$ giving us

$$
\overline{L_{\mathbb{C}}(E)_{n} \cdot L_{\mathbb{C}}(E)_{-n}} \subseteq \overline{C^{*}(E)_{n} \cdot C^{*}(E)_{-n}} .
$$

It remains to show that

$$
\overline{C^{*}(E)_{n} \cdot C^{*}(E)_{-n}} \subseteq \overline{L_{\mathbb{C}}(E)_{n} \cdot L_{\mathbb{C}}(E)_{-n}}
$$

It suffices to show

$$
C^{*}(E)_{n} \cdot C^{*}(E)_{-n}=\overline{L_{\mathbb{C}}(E)_{n}} \cdot \overline{L_{\mathbb{C}}(E)_{-n}} \subseteq \overline{L_{\mathbb{C}}(E)_{n} \cdot L_{\mathbb{C}}(E)_{-n}}
$$

To see this fix sequences $\left\{a_{i}\right\} \subseteq L_{\mathbb{C}}(E)_{n}$ and $\left\{b_{i}\right\} \subseteq L_{\mathbb{C}}(E)_{-n}$ with $a_{i} \rightarrow a$ and $b_{i} \rightarrow b$ as $i \rightarrow \infty$. By the algebra of limits we have $a_{i} b_{i} \rightarrow a b$ as $i \rightarrow \infty$. Thus $\left\{a_{i} b_{i}\right\}$ is a convergent sequence of $L_{\mathbb{C}}(E)_{n} \cdot L_{\mathbb{C}}(E)_{-n}$ and

$$
\overline{C^{*}(E)_{n} \cdot C^{*}(E)_{-n}}=\overline{L_{\mathbb{C}}(E)_{n} \cdot L_{\mathbb{C}}(E)_{-n}}
$$

Proof of Proposition 4.0.5: Suppose $L_{\mathbb{C}}(E)$ is not strongly graded. By [3, Lemma 2.1] there exists $n \in \mathbb{Z}$ such that

$$
L_{\mathbb{C}}(E)_{n} \cdot L_{\mathbb{C}}(E)_{-n} \subsetneq L_{\mathbb{C}}(E)_{0}
$$

Hence there exists $a \in L_{\mathbb{C}}(E)_{0}$ such that $a \notin L_{\mathbb{C}}(E)_{n} \cdot L_{\mathbb{C}}(E)_{-n}$. Recall that $C^{*}(E)_{0}=C^{*}(E)^{\gamma}$. We will show that $a \in C^{*}(E)^{\gamma} \backslash \overline{C^{*}(E)_{n} \cdot C^{*}(E)_{-n}}$, which would imply that $C^{*}(E)^{\gamma} \neq \overline{C^{*}(E)_{n} \cdot C^{*}(E)_{-n}}$ so $C^{*}(E)$ is not strongly graded.

Since $a \in L_{\mathbb{C}}(E)_{0}$ we have $a \in C^{*}(E)^{\gamma}$. By Lemma 4.0.7

$$
\overline{L_{\mathbb{C}}(E)_{n} \cdot L_{\mathbb{C}}(E)_{-n}}=\overline{C^{*}(E)_{n} \cdot C^{*}(E)_{-n}}
$$

so it suffices to show that $a \notin \overline{L_{\mathbb{C}}(E)_{n} \cdot L_{\mathbb{C}}(E)_{-n}}$. Recall that by Equation (3.4)

$$
L_{\mathbb{C}}(E)_{0}=\bigcup_{k} \mathcal{G}_{k}=\bigcup_{k}\left(\bigoplus_{v \in E^{0}} \mathcal{G}_{k}(v)\right)
$$


So there exists some $k$ and a finite collection of vertices $F \in E^{0}$ such that $a \in \bigoplus_{v \in F} \mathcal{G}_{k}(v)$ which we will denote $A_{F, k}$. Since $A_{F, k}$ is a finite direct sum of matricial algebras it is finite dimensional and hence a closed * subalgebra in $C^{*}(E)$.

By Lemma 4.0.6 $L_{\mathbb{C}}(E)_{n} \cdot L_{\mathbb{C}}(E)_{-n}$ is a *-subalgebra so the intersection $A_{F, k} \cap L_{\mathbb{C}}(E)_{n} \cdot L_{\mathbb{C}}(E)_{-n}$ will be a closed *-subalgebra of $A_{F, k}$. Since

$$
a \notin A_{F, k} \cap L_{\mathbb{C}}(E)_{n} \cdot L_{\mathbb{C}}(E)_{-n},
$$

there exists an open set $U_{a}$ in $A_{F, k}$, with respect to the subspace topology, containing $a$ such that

$$
U_{a} \cap\left(A_{F, k} \cap L_{\mathbb{C}}(E)_{n} \cdot L_{\mathbb{C}}(E)_{-n}\right)=\emptyset .
$$

Since $U_{a} \cap A_{F, k}=U_{a}$, we have $U_{a} \cap L_{\mathbb{C}}(E)_{n} \cdot L_{\mathbb{C}}(E)_{-n}=\emptyset$. Thus

$$
a \notin \overline{L_{\mathbb{C}}(E)_{n} \cdot L_{\mathbb{C}}(E)_{-n}} A_{F, k}
$$

where closure is taking place in $A_{F, k}$. But, $A_{F, k}$ is closed in $C^{*}(E)$, so by [8, Theorem 17.3]

$$
\overline{L_{\mathbb{C}}(E)_{n} \cdot L_{\mathbb{C}}(E)_{-n}} A_{F, k}=\overline{L_{\mathbb{C}}(E)_{n} \cdot L_{\mathbb{C}}(E)_{-n}}
$$

in $C^{*}(E)$. Hence $a \notin \overline{L_{\mathbb{C}}(E)_{n} \cdot L_{\mathbb{C}}(E)_{-n}}$ as needed.

Theorem 4.0.3 follows from Proposition 4.0.4 and Proposition 4.0.5.

Proof of Theorem 4.0.2: Let $E$ be a row-finite graph. Then $C^{*}(E)$ is a strongly graded $C^{*}$-algebra if and only if $L_{\mathbb{C}}(E)$ is a strongly graded ring by Theorem 4.0.3. Now $L_{\mathbb{C}}(E)$ is a strongly graded ring if and only if $E$ has no sources and satisfies Condition (Y) by [3, Theorem 4.1]. This completes the proof. 


\section{Bibliography}

[1] Abrams, G., ARA, P., And Molina, M. S. Leavitt Path Algebras. Springer, Nov. 2017.

[2] BRIDGeS, D. S. Foundations of Real and Abstract Analysis. Graduate Texts in Mathematics. Springer-Verlag, New York, 1998.

[3] Clark, L. O., Hazrat, R., AND Rigby, S. W. Strongly graded groupoids and strongly graded Steinberg algebras. arXiv:1711.04904 [math] (Nov. 2017).

[4] DADE, E. C. Group-graded rings and modules. Mathematische Zeitschrift 174, 3 (Oct. 1980), 241-262.

[5] Exel, R. Partial Dynamical Systems, Fell Bundles and Applications, vol. 224 of Mathematical Surveys and Monographs. American Mathematical Society, Sept. 2017.

[6] Hungerford, T. W. Algebra /[by] Thomas W. Hungerford. Holt, Rinehart and Winston, New York, 1974.

[7] MaC Lane, S. Algebra / [by] Saunders MacLane [and] Garrett Birkhoff. Macmillan, New York, 1967.

[8] Munkres, J. Topology, 2 edition ed. Pearson, Upper Saddle River, NJ, Jan. 2000. 
[9] Murphy, G. J. C*-Algebras and Operator Theory, 1 edition ed. Academic Press, Boston, Sept. 1990.

[10] Raeburn, I. Graph Algebras. Conference Board of the Mathematical Sciences, 2005.

[11] RAEBURN, I. Deformations of Fell bundles and twisted graph algebras. Mathematical Proceedings of the Cambridge Philosophical Society 161, 03 (Nov. 2016), 535-558.

[12] RAEBURN, I. On graded $C^{*}$-algebras. Bulletin of the Australian Mathematical Society 97 (Oct. 2017), 1-6.

[13] Raeburn, I., AND Williams, D. P. Morita Equivalence and Continuous-trace $C^{*}$-algebras. American Mathematical Soc., 1998.

[14] TOMFORDE, M. Leavitt path algebras with coefficients in a commutative ring. Journal of Pure and Applied Algebra 215, 4 (Apr. 2011), 471484. 